\title{
Nuclear pore protein NUP88 activates anaphase- promoting complex to promote aneuploidy
}

\author{
Ryan M. Naylor, ${ }^{1}$ Karthik B. Jeganathan, ${ }^{2}$ Xiuqi Cao, ${ }^{2}$ and Jan M. van Deursen ${ }^{1,2}$ \\ 'Department of Biochemistry and Molecular Biology and 'Department of Pediatric and Adolescent Medicine, Mayo Clinic, Rochester, Minnesota, USA.
}

\begin{abstract}
The nuclear pore complex protein NUP88 is frequently elevated in aggressive human cancers and correlates with reduced patient survival; however, it is unclear whether and how NUP88 overexpression drives tumorigenesis. Here, we show that mice overexpressing NUP88 are cancer prone and form intestinal tumors. To determine whether overexpression of NUP88 drives tumorigenesis, we engineered transgenic mice with doxycycline-inducible expression of Nup88. Surprisingly, NUP88 overexpression did not alter global nuclear transport, but was a potent inducer of aneuploidy and chromosomal instability. We determined that NUP88 and the nuclear transport factors NUP98 and RAE1 comprise a regulatory network that inhibits premitotic activity of the anaphase-promoting complex/cyclosome (APC/C). When overexpressed, NUP88 sequesters NUP98-RAE1 away from APC/ $\mathrm{C}^{\mathrm{CDH} 1}$, triggering proteolysis of polo-like kinase 1 (PLK1), a tumor suppressor and multitasking mitotic kinase. Premitotic destruction of PLK1 disrupts centrosome separation, causing mitotic spindle asymmetry, merotelic microtubule-kinetochore attachments, lagging chromosomes, and aneuploidy. These effects were replicated by PLK1 insufficiency, indicating that PLK1 is responsible for the mitotic defects associated with NUP88 overexpression. These findings demonstrate that the NUP88-NUP98-RAE1-APC/ $\mathrm{C}^{\mathrm{CDH} 1}$ axis contributes to aneuploidy and suggest that it may be deregulated in the initiating stages of a broad spectrum of human cancers.
\end{abstract}

\section{Introduction}

Nuclear pore complexes (NPCs) are channels embedded in the nuclear envelope (NE) that facilitate trafficking of macromolecules into and out of the nucleus (1). The NPC consists of approximately 30 distinct proteins (nucleoporins), each present in multiple copies. Although significant progress has been made in understanding how the NPC is structurally organized, the precise cellular functions of many individual nucleoporins remain unclear. Because of the remarkable complexity and importance of nucleocytoplasmic transport in maintaining proper cellular function, defects in this process have long been suspected to be important contributors to disease. However, it has been difficult to test this idea in vivo due to the essential nature of most transport components.

The most direct link between the transport machinery and human disease comes from the discovery of cancer-associated recurrent chromosomal translocations in various nucleoporin-encoding genes including TPR, NUP358 (RANBP2), NUP98, and NUP214 (CAN) (1). However, the association between another NPC component, NUP88, and cancer is unique in that NUP88 is commonly overexpressed in several malignant neoplasms $(1,2)$. By virtue of its overexpression, NUP88 has been proposed to be a clinical prognosticator. Indeed, the extent to which NUP88 is overexpressed has been shown to correlate with tumor aggressiveness and reduced patient survival in some neoplastic diseases $(1,3)$. Despite these observations, whether and how NUP88 overexpression drives malignant transformation and tumorigenesis remains unknown.

Conflict of interest: The authors have declared that no conflict of interest exists. Submitted: April 9, 2015; Accepted: November 13, 2015.

Reference information: J Clin Invest. 2016;126(2):543-559. doi:10.1172/JCI82277.
NUP88 is a non-phenylalanine-glycine (FG) repeat containing nucleoporin that links NUP214 to the cytoplasmic face of the NPC (1). NUP214 is an essential protein that contains an FG-repeat motif and directly interacts with CRM1, the main export receptor for nuclear export signal-containing (NES-containing) cargos (1). NUP88 also interacts with NUP98, which itself is in a dynamic subcomplex with the mRNA export factor RAE1 (1). However, whether a NUP88-NUP98-RAE1 subcomplex exists and what its functional and physiological relevance might be have not been established.

In HeLa cells, altered expression of NUP88 has been associated with multinucleation and centrosome amplification, linking NUP88 and mitosis through an unknown mechanism (4). These observations are in alignment with the broader emerging theme that nuclear transport factors play key roles in regulating chromosome segregation following NE breakdown (NEBD) and NPC disassembly early in mitosis (5). The close connection between the nuclear transport machinery and mitosis is further highlighted by the observation that NPC-bound MAD1-MAD2 heterodimers catalyze the formation of mitotic checkpoint complexes (MCCs) during interphase, much like they do when they are bound to unattached kinetochores in mitosis (6). The MCC consists of MAD2, BUBR1, BUB3, and the anaphase-promoting complex/ cyclosome (APC/C) coactivator CDC20. The goal of the MCC is to block premature separase-induced sister chromatid separation and anaphase onset by inhibiting $\mathrm{APC} / \mathrm{C}^{\mathrm{CDC} 20}$-mediated proteolysis of securin and cyclin B1 until each chromosome is bioriented on the metaphase plate (7). Attachment of the last kinetochore to the mitotic spindle releases CDC20 from the MCC, allowing it to bind and activate the E3 ubiquitin ligase activity of the APC/C. Following anaphase onset, $\mathrm{CDH} 1$ replaces CDC2O as the APC/C 
coactivator to orchestrate the ordered proteolysis of key mitotic kinases, including PLK1 and aurora A and B (8).

In contrast to $\mathrm{APC} / \mathrm{C}^{\mathrm{CDC} 20}$ regulation, there are several mechanisms of $\mathrm{APC} / \mathrm{C}^{\mathrm{CDH} 1}$ inhibition that cooperate throughout the cell cycle. The most well-established mechanism is CDK-mediated phosphorylation of $\mathrm{CDH} 1$, which prevents $\mathrm{CDH} 1$ from binding to the APC/C from $G_{1} / S$ until the metaphase-to-anaphase transition (9). CDH1 dephosphorylation is achieved in late metaphase as CDC14 phosphatase activity increases and cyclin B1 levels simultaneously decrease (9). APC/ $\mathrm{C}^{\mathrm{CDH} 1}$ is then active from anaphase until the subsequent $G_{1} / S$ transition, where its activity is quenched by a combination of at least four mechanisms (9): first, EMI1 binds and inactivates $\mathrm{APC} / \mathrm{C}^{\mathrm{CDH}}$; second, cyclins $\mathrm{A}$ and $\mathrm{E}$ accumulate in late $\mathrm{G}_{1}$ and mediate the CDK-dependent inhibitory phosphorylation of $\mathrm{CDH} 1$; third, the APC/C E2-conjugating enzyme Ubch10 is polyubiquitinated in an $\mathrm{APC} / \mathrm{C}^{\mathrm{CDH1}}$-mediated fashion and destroyed late in the $\mathrm{G}_{1}$ phase; fourth, $\mathrm{CDH} 1$ is thought to self-destruct at the $\mathrm{G}_{1} / \mathrm{S}$ transition through autoubiquitination.

Two additional mechanisms have been proposed to inhibit APC/C $\mathrm{C}^{\mathrm{CDH} 1}$ during early mitosis. First, MAD2L2 prevents CDH1 from interacting with the APC/C through direct protein-protein interaction (10). Second, the NUP98-RAE1 subcomplex has been shown to bind and inhibit residual $\mathrm{APC} / \mathrm{C}^{\mathrm{CDH} 1}$ in order to fully preserve securin until the metaphase-to-anaphase transition (11, 12). Although the latter mechanism is not widely accepted, independent groups have shown that the NUP98-RAE1 subcomplex coprecipitates with APC/C ${ }^{\text {CDH1 }}$ (11-13). Importantly, NUP98-RAE1 has also been shown to block the selective APC/ $\mathrm{C}^{\mathrm{CDH1}}$-mediated polyubiquitination of securin in in vitro ubiquitination assays (12). Arguing against this mechanism are the established view that $\mathrm{APC} / \mathrm{C}^{\mathrm{CDC} 20}$, not $\mathrm{APC} / \mathrm{C}^{\mathrm{CDH} 1}$, is responsible for polyubiquitinating both cyclin $\mathrm{B} 1$ and securin in metaphase and the fact that neither NUP98 nor RAE1 was identified in a genome-wide RNAi screen to uncover spindle assembly checkpoint (SAC) components (14). Additional research is therefore needed to better understand the role of NUP98-RAE1 in regulating $\mathrm{APC} / \mathrm{C}^{\mathrm{CDH}}$.

The studies presented here originated from the key open question as to whether NUP88 overexpression is a cause or consequence of tumorigenesis. Using a newly engineered mouse strain, we demonstrate that NUP88 overexpression drives tumor initiation in tumor types for which it serves as a poor clinical prognosticator. Rather than altering global nuclear transport, we found that high levels of NUP88 promoted aneuploidy and chromosomal instability (CIN) through the premitotic degradation of PLK1, a tumor suppressor and mitotic kinase. Our data show that the NUP98-RAE1 complex is normally responsible for inhibiting the premitotic activity of $\mathrm{APC} / \mathrm{C}^{\mathrm{CDH} 1}$ but that NUP88 sequesters NUP98-RAE1 away from $\mathrm{APC} / \mathrm{C}^{\mathrm{CDH} 1}$ when overexpressed, thereby promoting the unscheduled proteolysis of PLK1 and chromosome missegregation. Therefore, deregulation of the NUP88-NUP98-RAE1-APC/C ${ }^{\mathrm{CDH} 1}$ axis may be an important tumor-initiating event in human cancers.

\section{Results}

NUP88 overexpression drives tumorigenesis. To examine whether NUP88 overexpression drives tumorigenesis, we engineered doxycycline-inducibile (dox-inducible) Nup 88 -transgenic $\left(N u p 88^{T}\right)$ mice using site-directed integration of $\mathrm{KH} 2$ embryonic stem (ES) cells (15) and obtained two independent lines (Nup88 $8^{T 11}$ and $N u p 88^{T 13}$ ). In the presence of dox, mouse embryonic fibroblasts (MEFs) from both lines expressed approximately 4-fold more NUP88 than did control MEFs containing the reverse tetracycline-controlled transactivator (TA) but lacking the HA-Nup88 transgene (Supplemental Figure 1A; supplemental material available online with this article; doi:10.1172/JCI82277DS1). This level of overexpression is similar to that documented in several malignant neoplasms from patients (16). Additionally, induction of HA-NUP88 did not alter the expression of other nucleoporins (Supplemental Figure 1B). This is important, because elevated NUP88 protein levels in tumor samples is specific for NUP88 and does not represent a global increase in NPCs (2). HA-NUP88 properly integrated into nuclear pores and interacted with NUP214, NUP98, and RAE1 (Figure 1A and Supplemental Figure 1C), demonstrating that transgenic HA-NUP88 and endogenous NUP88 have similar biochemical properties. NUP88 accumulated in the cytoplasm of transgenic MEFs but was not elevated at the NE (Figure 1A and Supplemental Figure 1D), which is consistent with cytoplasmic accumulation of NUP88 in cancer cells (2). When administered dox-treated water (dox water), Nup $88^{T 11}$ and $N u p 88^{T 13}$ mice expressed substantially higher levels of NUP88 than did control mice in a broad spectrum of tissues (Figure 1B and Supplemental Figure 1E). Nup $88^{T 11}$ and $N u p 88^{T 13}$ mice did not express HA-NUP88 in the absence of dox, showing that transgene expression is tightly controlled in vivo (Supplemental Figure 1F). In contrast, some transgene leakiness was observed in MEFs cultured without dox (Supplemental Figure 1, A, B, and G), which prompted us to use MEFs without the HA-Nup88 transgene (TA MEFs) as control cells in all in vitro experiments. Collectively, the above data suggest that our newly generated transgenic mouse model recapitulates key features of NUP88 overexpression in human cancers.

Next, we established cohorts of Nup $88^{T}$ and TA mice on a mixed genetic background that were continuously administered dox water beginning at weaning age. At 14 months of age, the mice were sacrificed and screened for spontaneous tumors. We found that $N u p 88^{T}$ mice were markedly prone to tumors, with $56 \%$ of transgenic mice having at least one neoplastic lesion compared with $21 \%$ of control mice (Figure 1C). Mice overexpressing NUP88 were particularly prone to lung tumors (Figure 1, D and E), a tumor type that often has high NUP88 levels in patients (2).

Elevated NUP88 expression predicts poor survival in a subset of colorectal cancer patients (3), which prompted us to test whether NUP88 overexpression might enhance the formation of intestinal tumors in $A P C^{+/ M i n}$ mice (17). As shown in Figure 1, F and G, NUP88 overexpression markedly increased the incidence of mice presenting with colon tumors but had no impact on tumor multiplicity or size (Figure 1, H and I). Notably, there was also no difference in the multiplicity of small intestinal polyps between the $N u p 88^{T}$ and control mice (Supplemental Figure 1, $\mathrm{H}$ and I). In a parallel cohort of Nup $88^{T 11} A P C^{+/ M i n}$ mice, in which dox was discontinued 30 days before sacrifice, the incidence of colon tumors was indistinguishable from that seen in $N u p 88^{T 11}$ $A P C^{+/ M i n}$ mice that were continuously administered dox (Figure $1 G$ ), indicating that NUP88 overexpression in this model of colon tumorigenesis is an initiating event. Taken together, these tumor studies demonstrate that high levels of NUP88 drive tumorigen- 
A

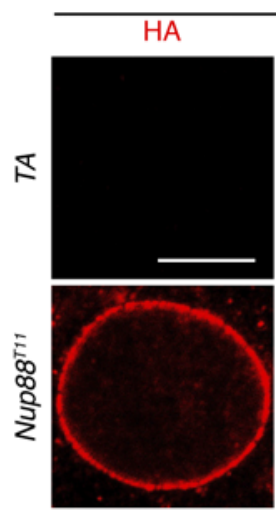

C

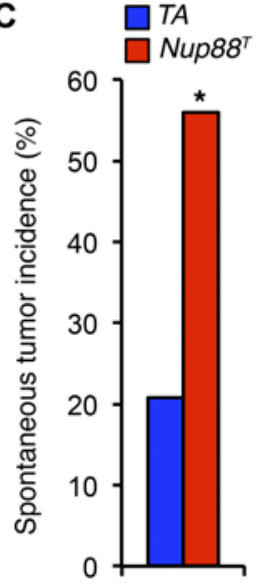

$\mathbf{F}$

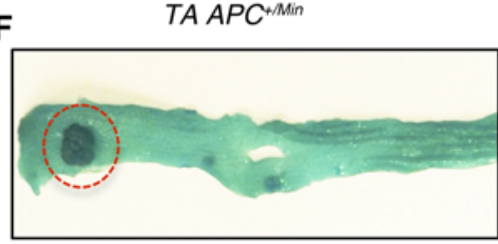

Nup88 ${ }^{\text {T11 }} A P C^{+M i n}$

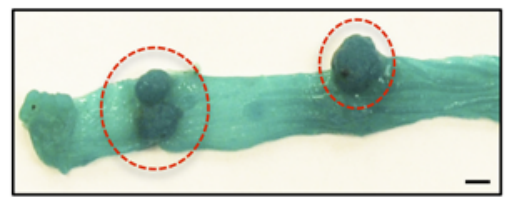

D
15 min 3\% PFA
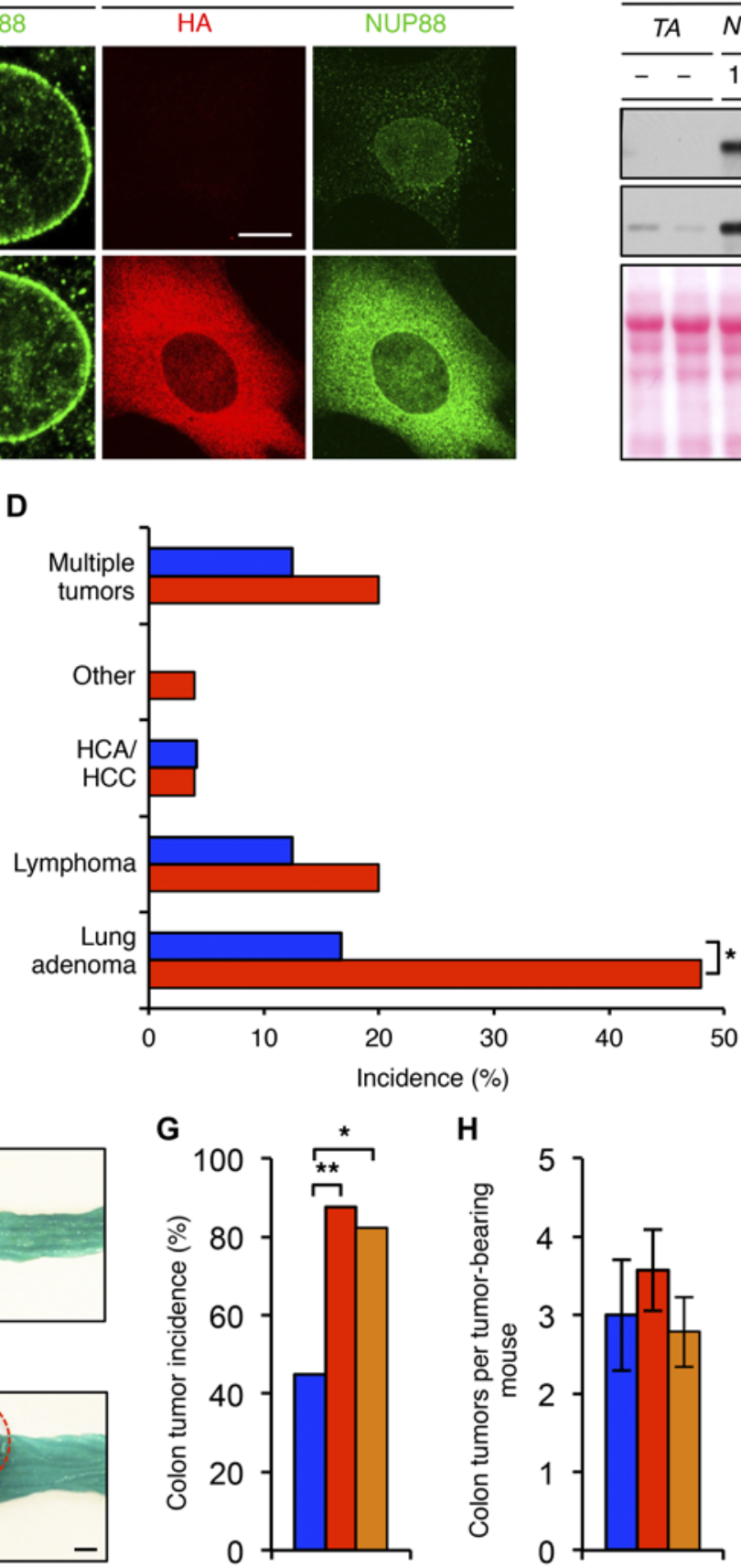

H

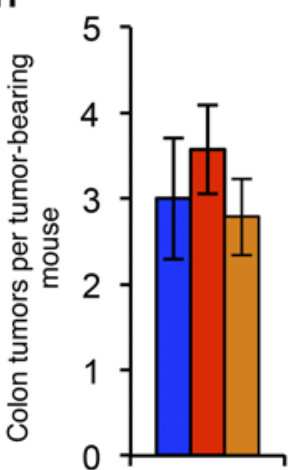

E

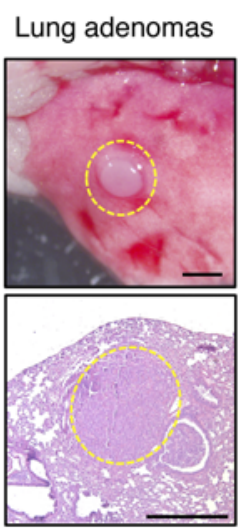

I

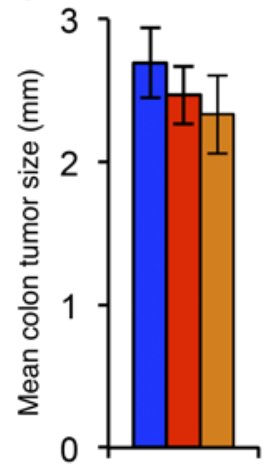

$T A A P C^{+/ M i n}(90 \mathrm{~d}+\mathrm{dox})$

Nup88 $8^{T 11} A P C^{+M i n}(90 \mathrm{~d}+\mathrm{dox})$

Nup88 ${ }^{T 11} A P C^{+/ M i n}(60 \mathrm{~d}+\mathrm{dox}, 30 \mathrm{~d}-\mathrm{dox})$

Figure 1. NUP88 overexpression drives tumorigenesis. (A) Representative images of MEFs immunostained for HA and NUP88. Fixation with PFA was optimized for the NE (5 minutes in 1\% PFA) or the entire cell (15 minutes in 3\% PFA). (B) Western blot analysis of lung and colon tissue lysates from 6-week-old dox-treated HA-Nup88 and control (TA) transgenic mice. Ponceau S (PonS) staining of blotted proteins served as a loading control. (C) Spontaneous tumor incidence in 14-month-old transgenic mice. Sample sizes of $24 T A$ and 25 Nup88 ${ }^{T}$ mice were used. (D) Spectrum of spontaneous tumor types observed. HCA, hepatocellular adenoma; HCC, hepatocellular carcinoma. (E) Representative gross and histological images of lung adenomas from 14-month-old transgenic mice. (F) Representative gross images of colon tumors from $T A A P C^{+/ M i n}$ and $N u p 88^{T 11} A P C^{+/ M i n}$ mice. (C) Colon tumor incidence, (H) multiplicity, and (I) mean colon tumor size in TA APC ${ }^{+/ M i n}$ and Nup8 $8^{T 11} A P C^{+/ M i n}$ mice. Sample sizes of $20 \mathrm{TA} A P C^{+/ M i n}(90 \mathrm{~d}+\mathrm{dox}), 24 \mathrm{Nup} 88^{T 11} A P C^{+/ M i n}(90$ $\mathrm{d}+\mathrm{dox})$, and $17 \mathrm{Nup} 88^{\mathrm{Th}} A P C^{+/ M i n}(60 \mathrm{~d}+\mathrm{dox}, 30 \mathrm{~d}$-dox) mice were used. Data represent the mean \pm SEM. Western blots are representative of 3 independent experiments. Statistical significance was determined using a 2-sided Fisher's exact test (C and $\mathbf{D})$ and a 1-sided Fisher's exact test with Bonferroni's correction (G). ${ }^{*} P<0.05$ and ${ }^{* *} P<0.01$. Scale bars: $10 \mu \mathrm{m}(\mathbf{A}), 1 \mathrm{~mm}(\mathbf{E}$ and $\mathbf{F})$. Nup88 ${ }^{T}$ indicates combined transgenic lines 11 and 13. 
A

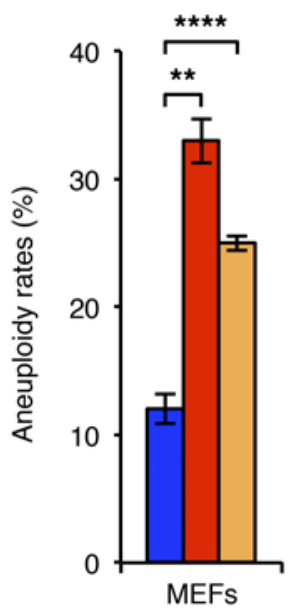

D
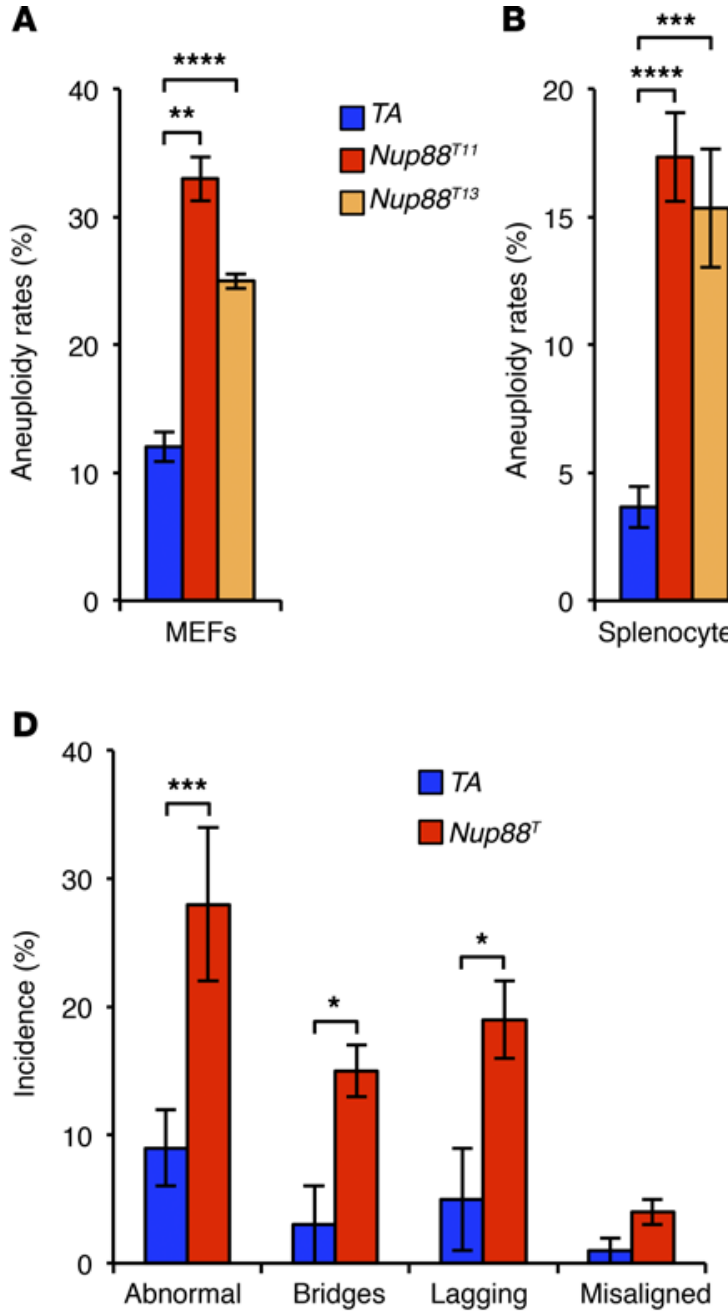

B
C

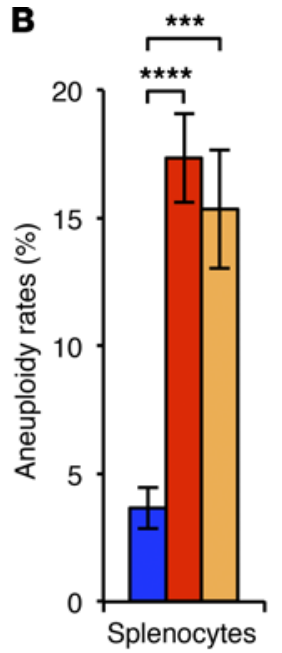

E

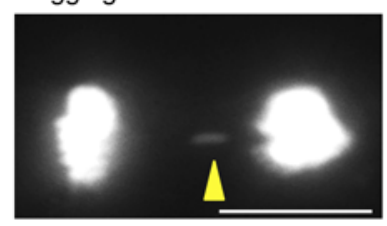

Bridge

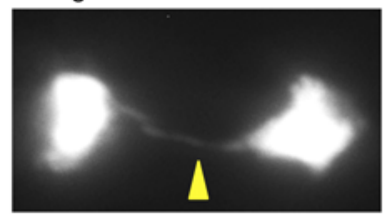

Mitotic Extrac

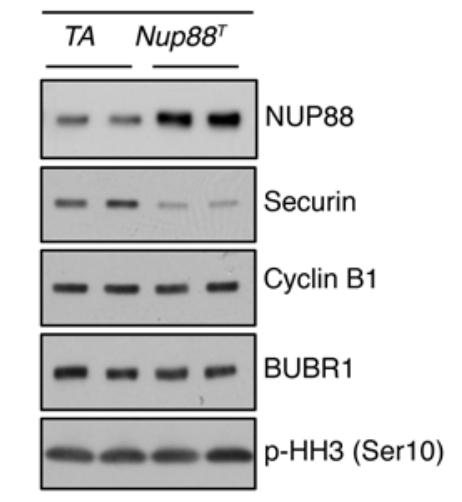

Figure 2. NUP88 overexpression mimics Nup98-Rae1 haploinsufficiency.

(A) Karyotypic analysis of numerical chromosomal abnormalities in $\mathrm{P} 5$

MEFs. (B) Same as in A for splenocytes harvested from 5-month-old mice. (C) Images of chromosome segregation errors observed using live-cell imaging. (D) Analysis of chromosome segregation errors in MEFs expressing H2B-mRFP from the indicated genotypes. (E) Western blot analysis of nocodazole-arrested MEFs. (F) Analysis of chromosome segregation errors in MEFs expressing H2B-mRFP from the indicated genotypes. ( $\mathbf{G}$ and $\mathbf{H}$ ) Analysis of spindle assembly checkpoint activity of MEFs challenged with nocodazole. Analyses in $\mathbf{A}$ and $\mathbf{B}$ were performed on 3 independent lines per genotype (50 spreads/ line). Analyses in $\mathbf{D}$ and $\mathbf{F}$ were performed on at least 3 independent lines per genotype ( 25 cells/line). Analyses in $\mathbf{G}$ and $\mathbf{H}$ were performed on at least 3 independent lines per genotype ( $>10$ cells/line). Data represent the mean \pm SEM. Western blots are representative of 3 independent experiments. Statistical significance was determined in $\mathbf{A}$ and $\mathbf{B}$ using 1-way ANOVA, followed by Tukey's multiple comparisons test. Statistical significance in $\mathbf{D}$ and $\mathbf{F}-\mathbf{H}$ was determined using a 2-tailed, unpaired $t$ test. ${ }^{*} P<0.05,{ }^{*} P<0.01,{ }^{* *} P<0.001$, and ${ }^{* * *} P<0.0001$. Scale bars: $10 \mu \mathrm{m}$. Nup $88^{T}$ indicates combined transgenic lines 11 and 13.

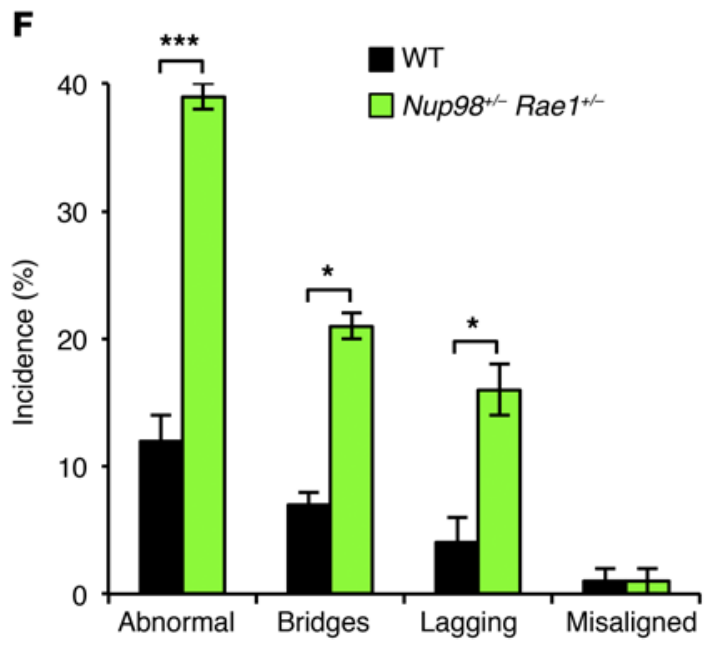

\section{$\mathbf{G}$}

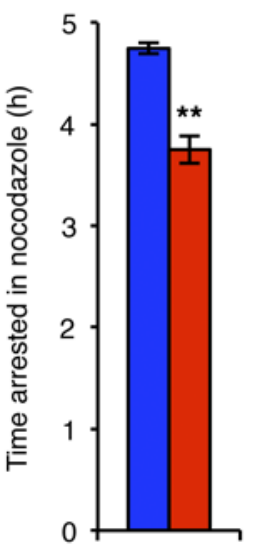

H

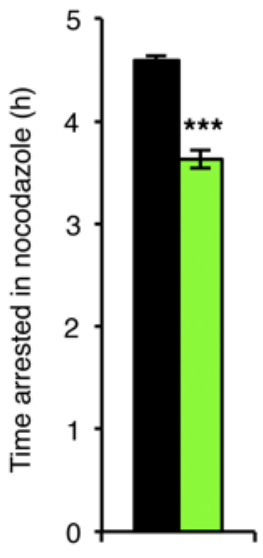

esis in multiple tissue types, including cancers for which it serves as a poor clinical prognosticator.

NUP88 overexpression causes chromosome missegregation and aneuploidy. To explore the mechanism(s) of oncogenesis, we first sought to determine whether NUP88 overexpression alters nucleocytoplasmic transport. However, transport assays for critical import and export pathways indicated that global macromolecular transport was unchanged in Nup88 $8^{T}$ MEFs (Supplemental Figure 2, A-H). This is perhaps not surprising, given that NUP88 accumulates in the cytoplasm rather than at NPCs when overexpressed (Figure 1A and Supplemental Figure 1D). Furthermore, the subcellular distribution of two cancer-critical proteins, p53 and nucleophosmin, were unchanged in MEFs overexpressing NUP88 (Supplemental Figure 2, I and J), supporting the notion that abnormal 
A
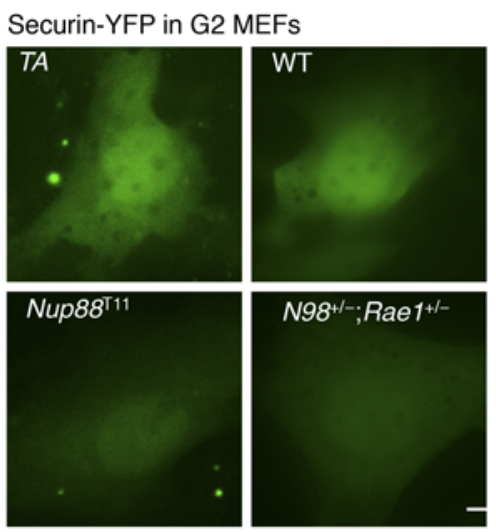

D $T A$

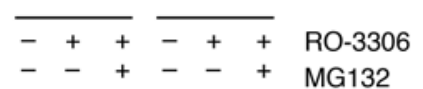

MG132

$\begin{array}{llllll}0 & 23 & 23 & 0 & 23 & 23 \\ \text { Time (h) }\end{array}$

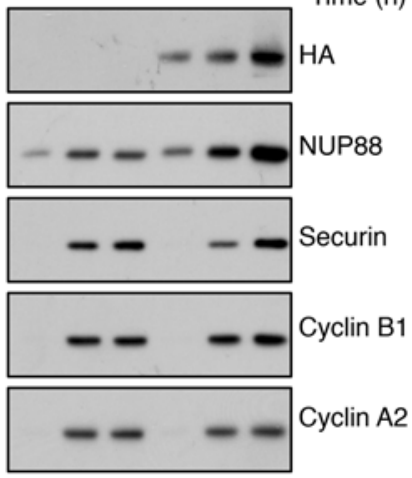

$-m-\infty-\infty$ Actin

E

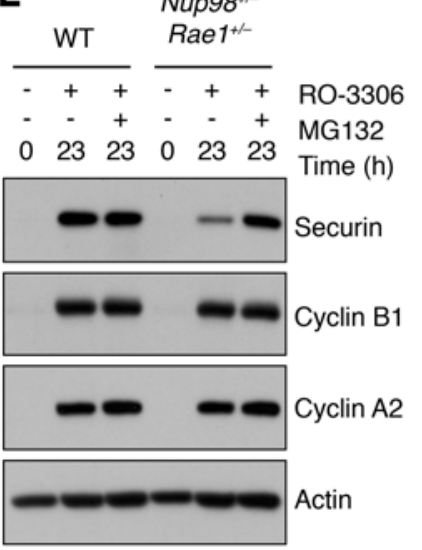

$\mathbf{F}$
B
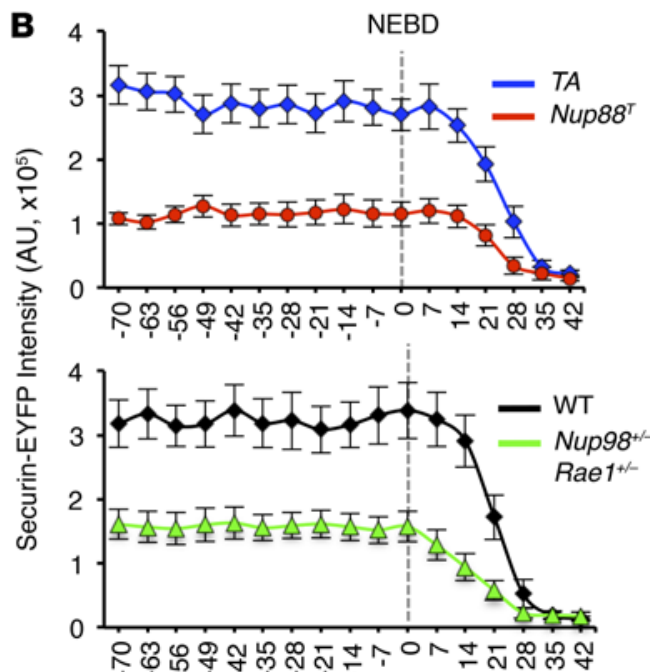

Time (min) Securin-YFP in G2 MEFs

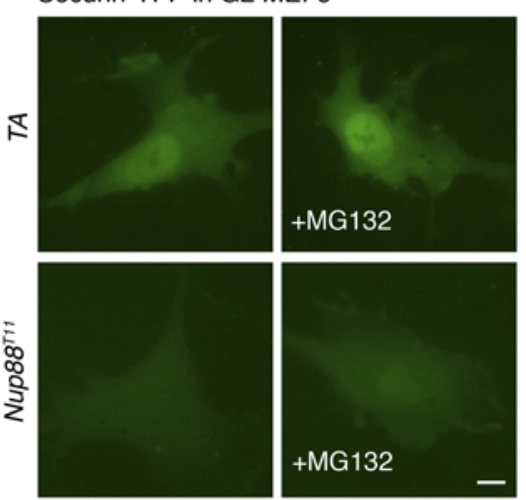

H

Securin-YFP in G2 MEFs
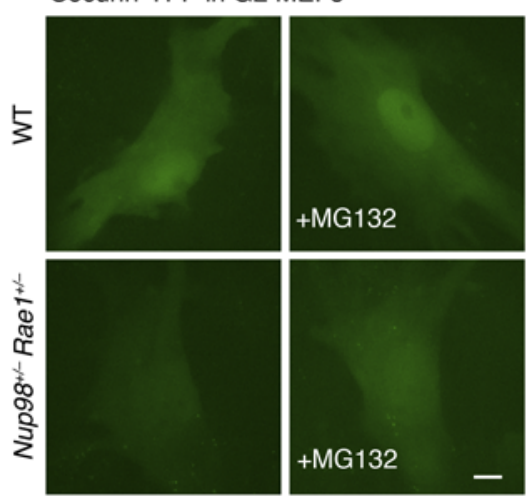

I
C

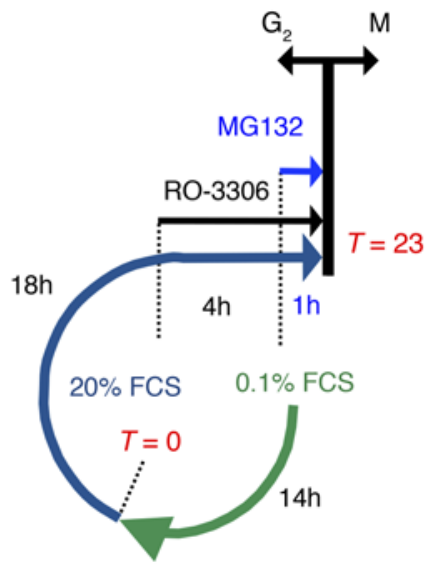

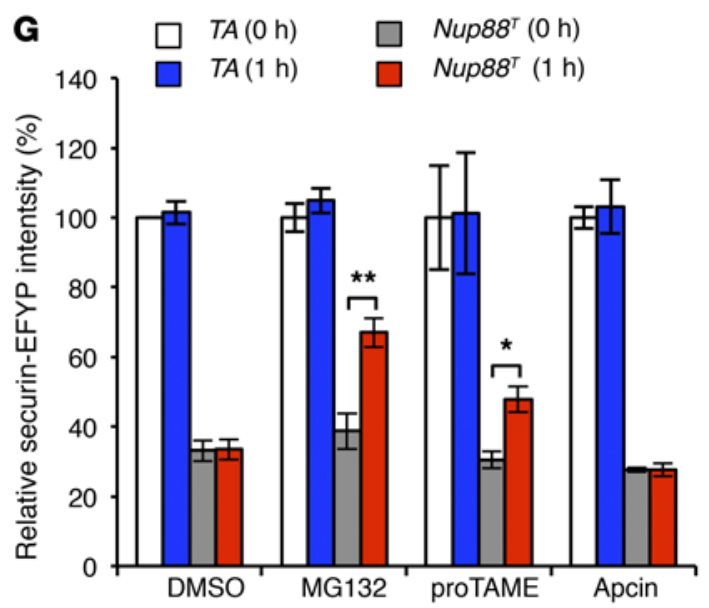

$\square \mathrm{WT}(0 \mathrm{~h})$

$\mathrm{N}^{2} 8^{+/-} \mathrm{Rae1}^{+/-}(\mathrm{O} \mathrm{h})$

WT (1 h)

N98 ${ }^{+/-}$Rae1 $^{*-}(1 \mathrm{~h})$

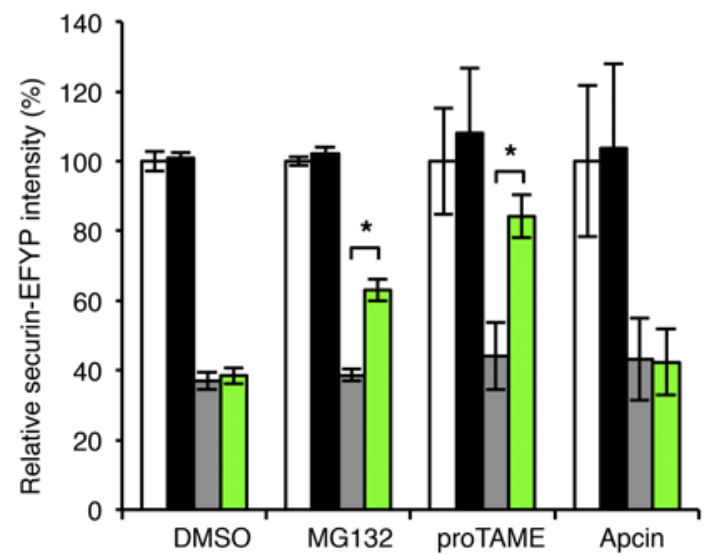

Figure 3. NUP98-RAE1 protects de novo synthesized securin from $A P C / C^{\mathrm{CDH} 1}$-mediated degradation in the $\mathrm{G}_{2}$ phase, and NUP88 overexpression perturbs this protective mechanism. (A) Images of $G_{2}$-phase MEFs of the indicated genotypes transduced with a lentivirus expressing securin-EYFP. (B) Quantification of securin-EYFP intensity in MEFs from $\mathbf{A}$ progressing from $\mathrm{G}_{2}$ through mitosis. (C) Strategy for $\mathrm{G}_{2}$-phase synchronization in MEFs. See Methods for more information. (D) Western blot analysis of Nup88 ${ }^{\top}$ MEFs synchronized in $G_{2}$ in the presence or absence of MG132. (E) Western blot analysis of Nup98 ${ }^{+/}$ Rae ${ }^{+/-}$MEFs synchronized in $G_{2}$ in the presence or absence of MG132. (F) Images of interphase Nup88 ${ }^{\top}$ MEFs expressing securin-EYFP before and after 1 hour of MC132 treatment. (C) Quantification of securin-EYFP intensity in MEFs from $\mathbf{F}$ treated with MG132, proTAME, or apcin. (H) Representative images of interphase Nup98+/- $\mathrm{Rae}^{++-}$MEFs expressing securin-EYFP before and after 1 hour of MG132 treatment. (I) Quantification of securin-EYFP intensity in MEFs from $\mathbf{H}$ treated with MG132, proTAME, or apcin. Analyses in B, G, and I were performed on 3 independent lines per genotype ( $>8$ cells/line). Data represent the mean \pm SEM. Western blots are representative of 3 independent experiments. Statistical significance in $\mathbf{G}$ and $\mathbf{I}$ was determined using a 2 -tailed, unpaired $t$ test. ${ }^{*} P<0.05$ and ${ }^{* *} P<0.01$. Scale bars: $10 \mu \mathrm{m}$. Nup88 ${ }^{T}$ indicates the combined transgenic lines 11 and 13 . 
A

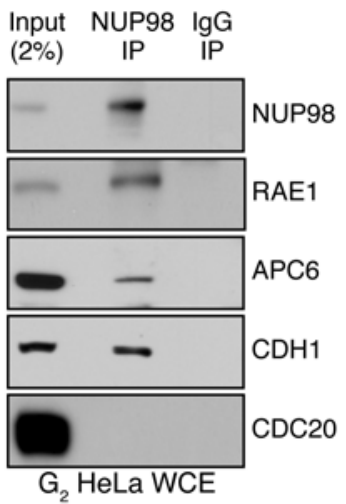

Input NUP98 Ig

D

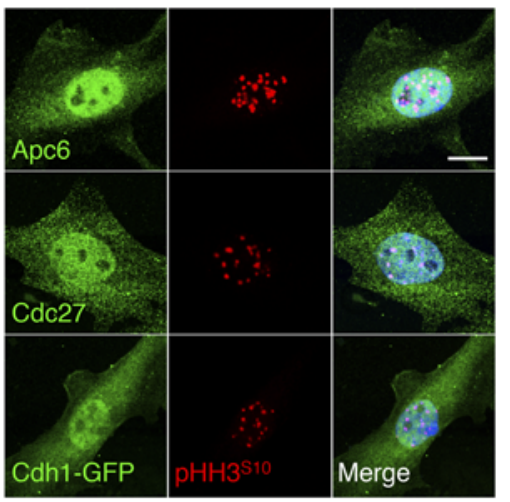

B

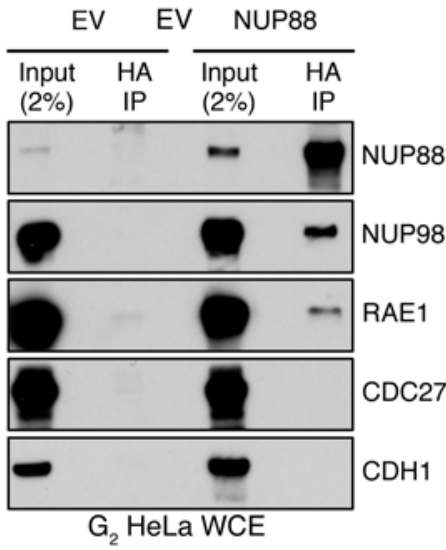

C

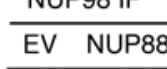

\begin{tabular}{|cc|}
\hline- & - \\
\hline 1.00 & 0.50
\end{tabular}

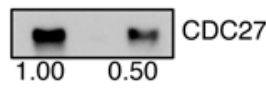
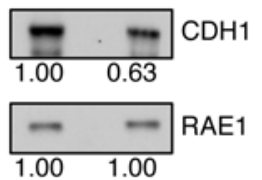

\begin{tabular}{|cc|}
\hline- & - \\
\hline 1.00 & 1.10
\end{tabular}
WCE

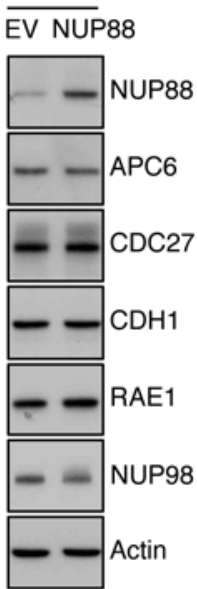

G

$\mathbf{F}$

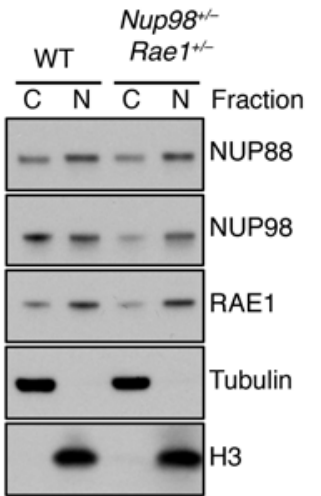

H

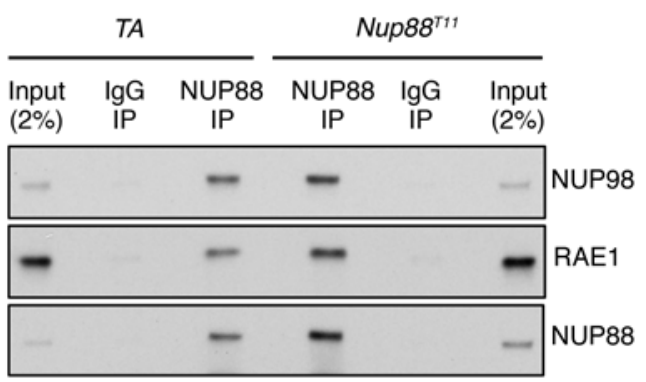

Cytoplasmic fraction
E

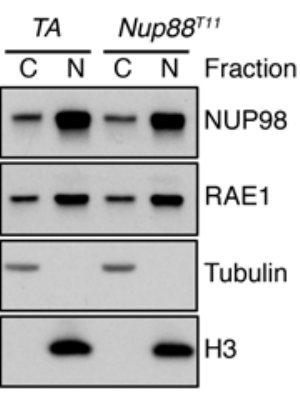

Nup98

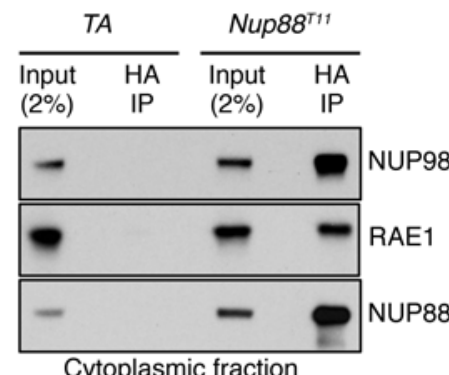

J

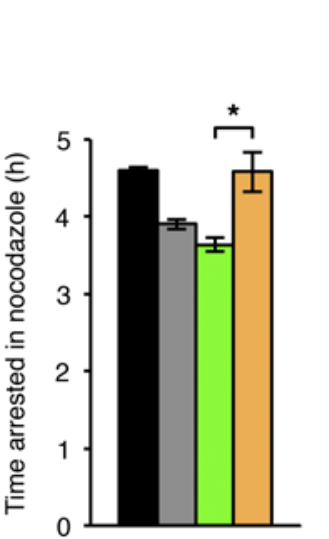

K Nup88

Nup98

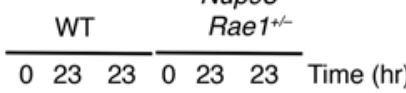

- + + - + + RO-3306

- $\ldots+\ldots$ + + MG132

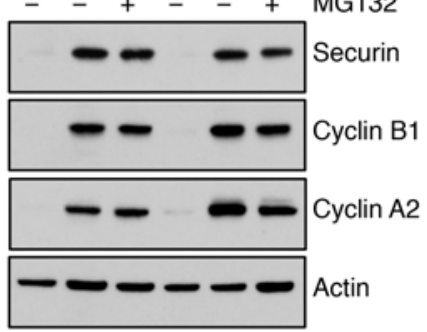

I $\stackrel{\text { a** }}{\star}$

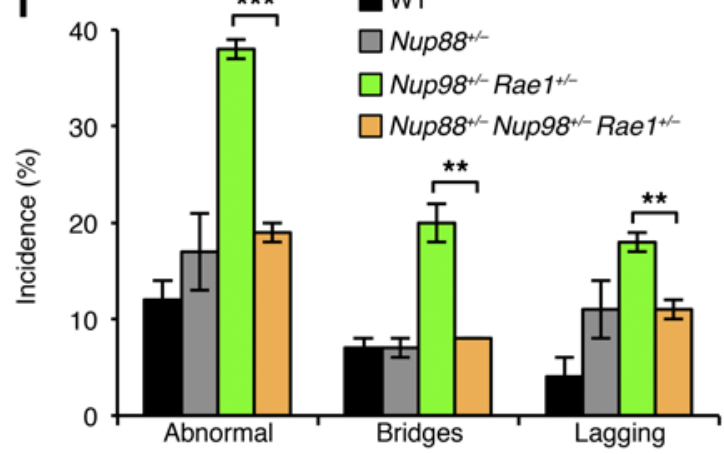

$\mathbf{L}$

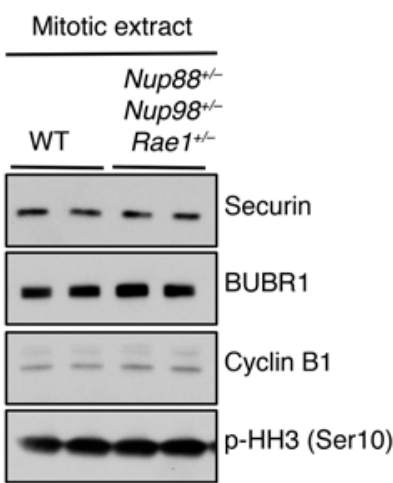


Figure 4. NUP88 sequesters NUP98-RAE1 away from APC/ $\mathrm{C}^{\mathrm{CDH} 1}$ prior to mitotic entry when overexpressed. (A) Coprecipitation analysis of R0-3306-treated HeLa cells immunoprecipitated with an anti-NUP98 Ab. (B) Coprecipitation analysis of R0-3306-treated HeLa cells transduced with pTRIPZ-HA-Nup88 (NUP88) or PTRIPZ empty vector (EV) lentiviruses and immunoprecipitated with anti-HA affinity matrix. (C) Coprecipitation analysis of cells in B immunoprecipitated with an anti-NUP98 Ab. Values represent NUP88/EV protein quantification ratios. (D) Images of WT MEFs immunostained for APC6, CDC27, or CDH1. p-HH3 (Ser10) was used to identify cells in the $\mathrm{G}_{2}$ phase. (E) Western blot analysis of cytoplasmic (C) and nuclear (N) fractions from MEFs of the indicated genotypes. (F) Same as in E. (C) Coprecipitation analysis of cytoplasmic fractions from MEFs of the indicated genotypes immunoprecipitated with anti-HA affinity matrix. (H) Same as in G, except immunoprecipitated with an anti-NUP88 Ab. (I) Live-cell-imaging analysis of chromosome segregation errors in H2B-mRFP-positive MEFs of the indicated genotypes. (J) Mitotic checkpoint analysis of MEFs in I challenged with nocodazole. (K) Western blot of RO-3306-treated or (L) nocodazole-arrested MEFs of the indicated genotypes. Analysis in I was performed on 3 independent lines per genotype (25 cells/line). Analysis in J was performed on 3 independent lines per genotype (>10 cells/ line). Data represent the mean \pm SEM. Western blots are representative of 3 independent experiments. Cyclin B1, cyclin A2, and actin blots in $\mathbf{K}$ and $\mathbf{L}$ are identical to those in Figure 5, G and H. WT and NUP98 RAE1 values in I and $\mathbf{J}$ are identical to those in Figure 2, F and $\mathrm{H}$. Statistical significance in $\mathbf{I}$ and $\mathrm{J}$ was determined using a 2-tailed, unpaired $t$ test. ${ }^{*} P<0.05$, ${ }^{*} P<0.01$, and ${ }^{* * *} P<0.001$. Scale bar: $10 \mu \mathrm{m}$. WCE, whole-cell extract.

nuclear transport is unlikely to contribute to tumor formation in Nup $88^{T}$ mice. Although it cannot be excluded that other proteins relevant to cancer may be aberrantly distributed in the context of NUP88 overexpression, the data obtained prompted us to explore defects in transport-independent mechanisms of neoplastic transformation that may be deregulated in Nup $88^{T}$ MEFs.

Given that several components of the nucleocytoplasmic transport machinery function in chromosome segregation during mitosis (5), we asked whether NUP88 overexpression resulted in aneuploidization. Indeed, $N u p 88^{T}$ MEFs had significantly higher aneuploidy rates than did control MEFs (Figure 2A and Supplemental Figure 3A). Consistently, splenocytes harvested from 5-month-old transgenic mice showed approximately 4 -fold higher rates of aneuploidy than did splenocytes from TA mice (Figure 2B and Supplemental Figure 3B). To determine the nature of the underlying chromosome segregation defects leading to aneuploidization, we followed primary MEFs expressing H2B-mRFP through unperturbed mitoses by live-cell imaging. We found that Nup $88^{T}$ MEFs had significantly higher rates of chromatin bridges and lagging chromosomes than did control MEFs (Figure 2, C and D). Earlier work in HeLa cells linked altered NUP88 expression to centrosome amplification and multipolar spindle formation (4); however, no such defects were observed in Nup $88^{T}$ MEFs (Supplemental Figure 4A). NUP88 overexpression also did not alter the timing of mitosis (Supplemental Figure 4B). Together, these data indicate that NUP88 overexpression promotes chromosome missegregation and aneuploidy.

Nup $88^{T}$ and Nup98 $98^{+-} \mathrm{Rae1}^{+/-} \mathrm{MEFs}$ have identical mitotic phenotypes. To understand how NUP88 overexpression induces chromosome segregation errors, we used Western blot analysis of mitotic Nup $88^{T}$ MEF lysates to screen for aberrant levels of key mitotic regulators. We found that BUBR1 and cyclin B1 were normally expressed (Figure 2E). Surprisingly, however, securin was expressed at markedly reduced levels.

Selective loss of securin was reminiscent of a previously reported mutant MEF line that had low levels of the nuclear transport proteins NUP98 and RAE1 (12). In this study, the NUP98RAE1 complex was found to bind and prevent $\mathrm{APC} / \mathrm{C}^{\mathrm{CDH}}$ from prematurely degrading securin during mitosis. Chromosome segregation analyses revealed that $\mathrm{Nup} 98^{+/-} \mathrm{Rae}^{+/-} \mathrm{MEFs}$, like $\mathrm{Nup} 88^{\mathrm{T}}$ MEFs, were prone to chromatin bridges and lagging chromosomes (Figure 2F). In addition, $\mathrm{Nup} 98^{+/-} \mathrm{Rael}^{+/-}$MEFs were reported to have a mild mitotic checkpoint defect when challenged with nocodazole (12). Side-by-side comparison of $N u p 88^{T}$ and $N u p 98^{+/-}$
$\mathrm{Rae1}^{+/-}$MEFs revealed mitotic checkpoint defects of equal magnitude (Figure 2, G and H), further demonstrating phenotypic similarity between the two mutants.

NUP98-RAE1 protects securin by inhibiting premitotic APC/C $C^{C D H 1}$ activity. Traditionally, MCC proteins like BUBR1 and MAD2 are thought to protect cyclin $\mathrm{B} 1$ and securin against $\mathrm{APC} / \mathrm{C}^{\mathrm{CDC} 20}$ mediated proteasome destruction beginning at NEBD and lasting until spindle biorientation (7). However, recent evidence indicates that the MCC inhibits APC/ $\mathrm{C}^{\mathrm{CDC} 20}$ during the $\mathrm{G}_{2}$ phase to allow for the accumulation of cyclin $\mathrm{B} 1$ prior to mitosis (6, 18). This prompted us to ask whether $\mathrm{APC} / \mathrm{C}^{\mathrm{CDH} 1}$ is also premitotically active and, if so, whether securin accumulation in the $\mathrm{G}_{2}$ phase requires a parallel mechanism whereby NUP98-RAE1 inhibits this activity. To address this question, we monitored Nup $88^{T}$ and $N u p 98^{+/-} \mathrm{Rae}^{1^{+/-}}$MEFs by live-cell imaging for their ability to accumulate securin-enhanced yellow fluorescent protein (EYFP) prior to mitosis. As shown in Figure 3, A and B, both MEF types entered mitosis with smaller amounts of securinEYFP than did their respective controls.

To obtain further evidence that securin is targeted for proteasome-mediated degradation during the $\mathrm{G}_{2}$ phase in $N u p 88^{T}$

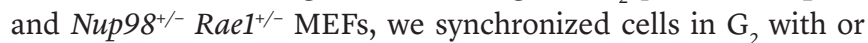
without the addition of MG132 and assessed securin protein levels (Figure 3, C-E). Using this approach, we found that $N u p 88^{T}$ and Nup98 ${ }^{+/-} \mathrm{Rael}^{+/-}$MEFs had low endogenous levels of securin in the $G_{2}$ phase (Figure 3, D and E). Furthermore, securin levels were restored upon addition of MG132, suggesting that securin is being actively degraded in a proteasome-dependent fashion prior to mitosis. In additional live-cell-imaging experiments, a substantial increase in securin-EYFP intensity was detected in

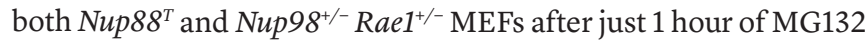
treatment (Figure 3, F-I). Because MG132 inhibits all proteasome degradation and does not specifically block the activity of APC/ $\mathrm{C}^{\mathrm{CDH}}$, we repeated these live-cell-imaging experiments with proTAME, a pan-APC/C inhibitor (19), or apcin, a CDC20-specific inhibitor (20). Consistent with the idea that $\mathrm{APC} / \mathrm{C}^{\mathrm{CDH} 1}$ is responsible for targeting securin for proteasome degradation in $\mathrm{G}_{2}$-phase Nup $88^{\mathrm{T}}$ and $\mathrm{Nup} 98^{+/-} \mathrm{Rael}^{+/-} \mathrm{MEFs}$, proTAME treatment increased securin-EYFP intensity, while apcin did not (Figure 3, G-I). In complementary experiments, neither MG132, nor proTAME, nor apcin changed endogenous cyclin B1 protein levels in Nup $88^{T}$ or Nup $98^{+/-} \mathrm{Rae}^{+/-}$MEFs (Supplemental Figure 5, A and B). As expected, both proTAME and apcin prevented unscheduled degradation of cyclin $\mathrm{B} 1$ in mutant $\mathrm{MEFs}$, in which $\mathrm{APC} / \mathrm{C}^{\mathrm{CDC} 20}$ 
A

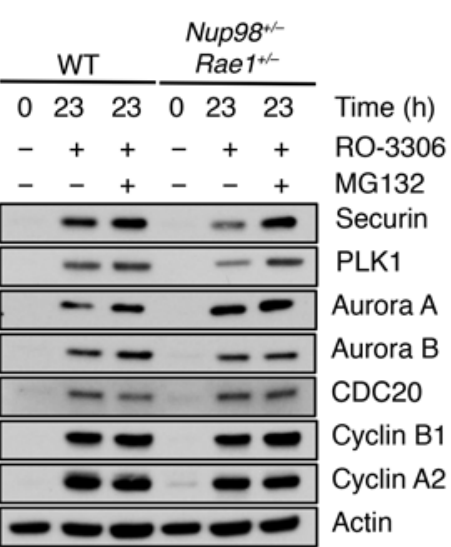

B

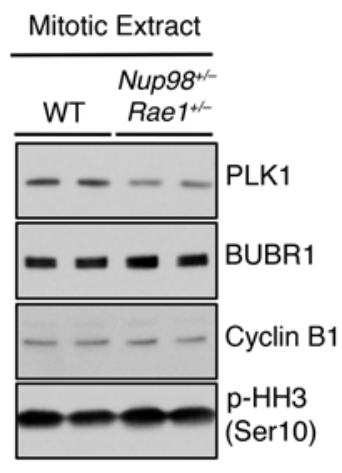

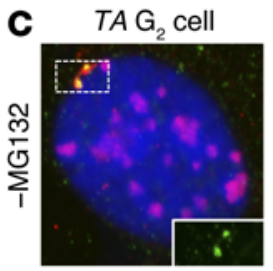

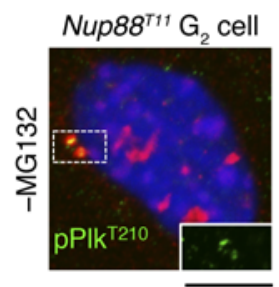

G

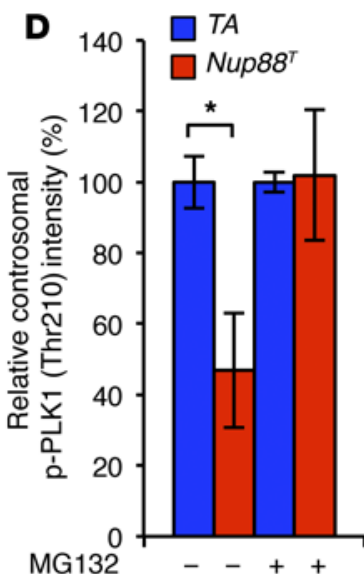

H

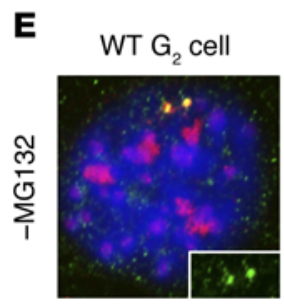

Nup98 ${ }^{\text {T11 }}$ Rae1 $^{+/-} \mathrm{G}_{2}$ cell

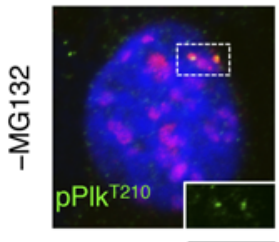

$\mathbf{F}$

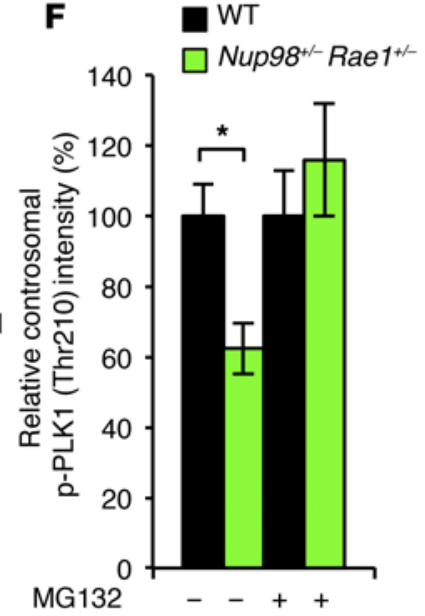

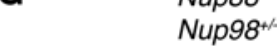

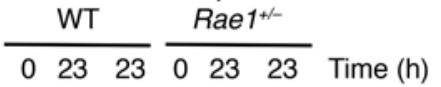

$-++-++\mathrm{RO}-3306$

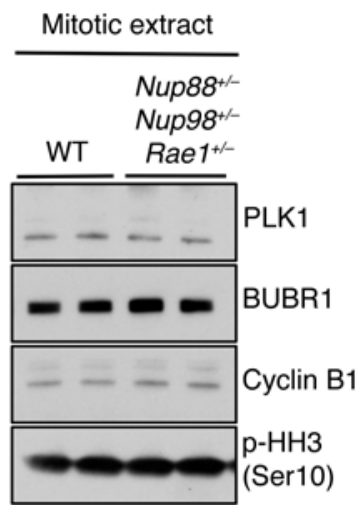

I

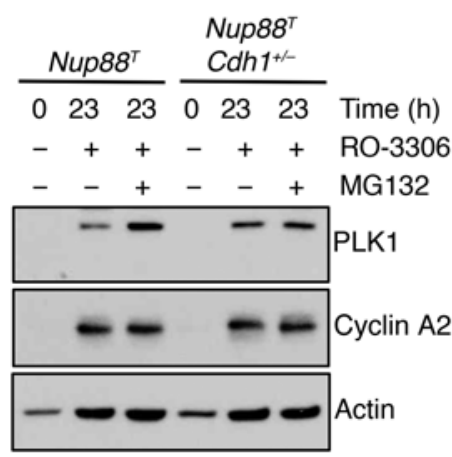

J

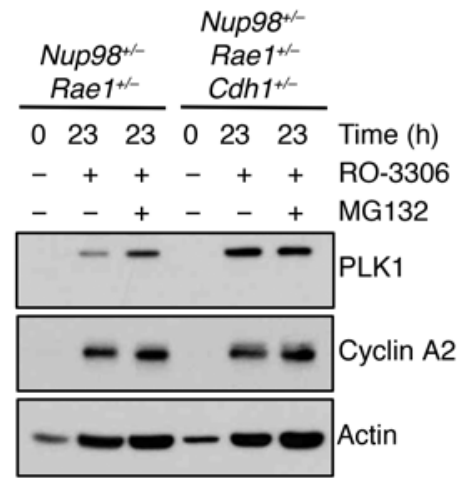

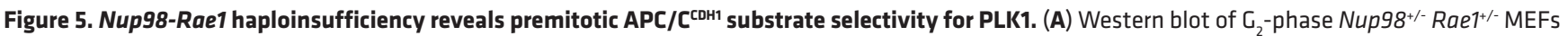
with or without MG132 treatment. (B) Western blot of nocodazole-arrested Nup98 $8^{+-}$Rae1+- MEFs. (C) Images of $\mathrm{G}_{2}$ MEFs of the indicated genotypes immunostained for p-PLK1 (Thr210), $\gamma$-tubulin, and p-HH3 (Ser10). $\gamma$-Tubulin and p-HH3 (Ser10) were distinguished by subcellular compartmentalization. (D) Quantification of centrosomal p-PLK1 (Thr210) intensity of cells in C with or without MG132 treatment. (E) Same as in C. (F) Same as in D. (G) Western blot from R0-3306-treated or (H) nocodazole-arrested MEFs of the indicated genotypes. (I and J) Same as in G. DNA in C and E was visualized with Hoechst. Analyses in $\mathbf{D}$ and $\mathbf{F}$ were performed on 3 independent lines per genotype (at least 20 cells/line). Data represent the mean \pm SEM. Western blots are representative of 3 independent experiments. Cyclin B1, cyclin A2, and actin blots from $\mathbf{G}$ and $\mathbf{H}$ are identical to those in Figure $4, \mathrm{~K}$ and $\mathrm{L}$. Statistical significance was determined in $\mathbf{D}$ and $\mathbf{F}$ using a 2-tailed, unpaired $t$ test. ${ }^{*} P<0.05$. Scale bars: $10 \mu \mathrm{m}$. Nup88 ${ }^{\top}$ indicates the combined transgenic lines 11 and 13.

was prematurely active due to BUBR1 insufficiency (Supplemental Figure 5C and ref. 18). Consistently, proTAME and apcin have been shown to stabilize cyclin B1 levels in a dose-dependent fashion by blocking $\mathrm{APC} / \mathrm{C}^{\mathrm{CDC} 20}(20)$.

A NUP88-NUP98-RAE1 axis regulates premitotic APC/C ${ }^{C D H 1}$ activity. These data suggest that $\mathrm{APC} / \mathrm{C}^{\mathrm{CDH}}$ must be inhibited by
NUP98-RAE1 during the $\mathrm{G}_{2}$ phase in order for securin to accumulate and demonstrate that NUP88 overexpression disables the protective effects of NUP98-RAE1. Given these results, we predicted that NUP88 sequesters NUP98-RAE1 away from APC/ $\mathrm{C}^{\mathrm{CDH} 1}$ in the $\mathrm{G}_{2}$ phase, thereby activating $\mathrm{APC} / \mathrm{C}^{\mathrm{CDH} 1}$ and triggering securin degradation. To test this model, we first asked whether 
A

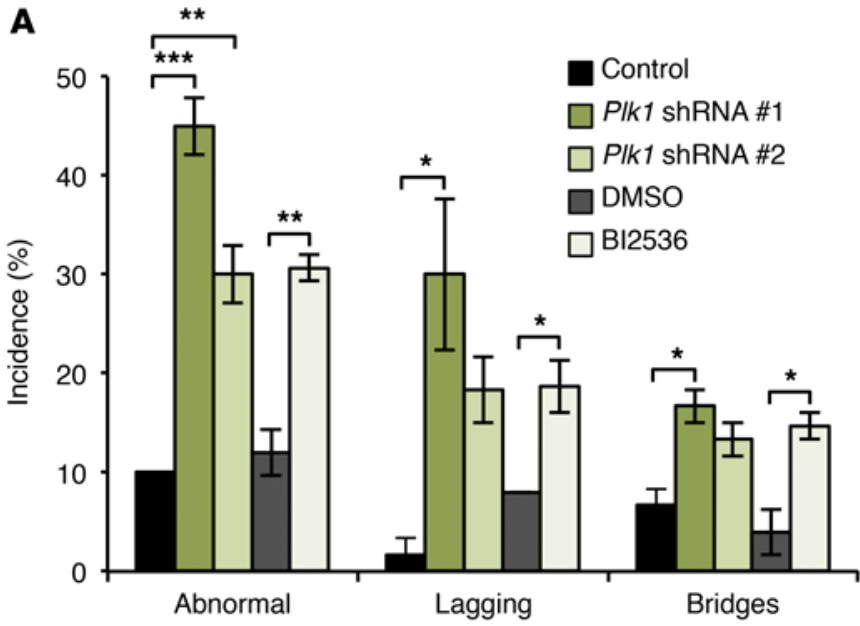

B

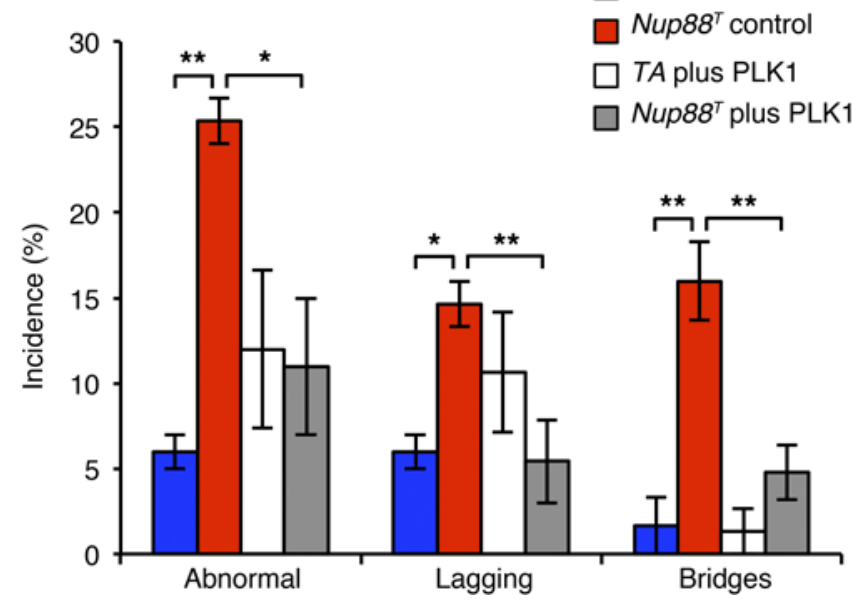

NUP98-RAE1 interacts with APC/C $\mathrm{C}^{\mathrm{CDH} 1}$ in whole-cell extracts from $\mathrm{G}_{2}$-phase HeLa cells. We chose this approach for two reasons: first, HeLa cells can be easily synchronized at the $G_{2} / M$ phase using the CDK1 inhibitor RO-3306 (21); and second, the pioneering work that demonstrated NUP98-RAE1-mediated inhibition of APC/ $\mathrm{C}^{\mathrm{CDH} 1}$ in mitosis was performed using HeLa cells (12). We found that premitotic NUP98-RAE1-APC/C $\mathrm{C}^{\mathrm{CDH} 1}$ complexes were readily identifiable (Figure 4A), supporting the notion that NUP98-RAE1 inhibits premitotic $\mathrm{APC} / \mathrm{C}^{\mathrm{CDH} 1}$ activity through a direct interaction. We then asked whether NUP88 interacts with NUP98-RAE1 independently of APC/C $\mathrm{C}^{\mathrm{CDH} 1}$. By expressing HA-Nup 88 in $\mathrm{G}_{2}$-phase HeLa cells, we were able to detect a NUP88-NUP98-RAE1 complex without APC/C components or CDH1 (Figure 4B). To determine whether NUP88 overexpression results in sequestration of NUP98-RAE1 away from APC/C ${ }^{\mathrm{CDH} 1}$ in the $\mathrm{G}_{2}$ phase, we immunoprecipitated NUP98 in HeLa cells overexpressing NUP88 and assessed the coprecipitation of various APC/C components and CDH1. As shown in Figure 4C and Supplemental Figure 6, NUP88 overexpression resulted in a reduction of NUP98-RAE1-APC/C $\mathrm{C}^{\mathrm{CDH}}$ inhibitory complexes by approximately $50 \%$.

Given that NUP88 accumulates in the cytoplasm rather than in NPCs when overexpressed (Figure 1A and Supplemental Figure 1D), we predicted that NUP98-RAE1-mediated inhibition of APC/ $\mathrm{C}^{\mathrm{CDH1}}$ occurs in the cytoplasm, away from NPCs. To test this, we
Figure 6. PLK1 insufficiency is responsible for the mitotic defects in Nup88 ${ }^{T}$ MEFs. (A) Chromosome missegregation analysis of H2B-mRFPpositive MEFs expressing an shRNA against Plk1 or treated with $100 \mathrm{nM}$ of the PLK1 inhibitor, BI2536. (B) Chromosome missegregation analysis of H2B-mRFP-positive MEFs overexpressing PLK1. Analyses in A and $\mathbf{B}$ were performed on at least 3 independent lines per genotype ( 25 cells/line). Data represent the mean \pm SEM. Statistical significance was determined in $\mathbf{A}$ using a 1-way ANOVA, followed by Tukey's multiple comparisons test to compare the control shRNA versus PIk1 shRNAs and a 2-tailed, unpaired $t$ test to compare DMSO versus BI2536 treatment. Statistical significance was determined in B using a 1-way ANOVA, followed by Tukey's multiple comparisons test. ${ }^{*} P<0.05$, ${ }^{* *} P<0.01$, and ${ }^{* * *} P<0.001$. Nup88 $8^{T}$ indicates the combined transgenic lines 11 and 13.

first sought to determine the subcellular localization of APC/C and its cofactor CDH1 (Figure 4D). Using immunofluorescence, we observed that the APC/C components CDC27 and APC6 were present in the cytoplasm and nucleus, albeit in greater amounts within the nucleus. Moreover, by expressing a CDH1-GFP fusion protein (22), we found that CDH1, like CDC27 and APC6, was also present in both subcellular compartments. We then sought to determine the localization of NUP98 and RAE1 by subcellular fractionation and compare the distribution of the NUP98-RAE1 complex between $N u p 88^{T}$ and control MEFs (Figure 4E). Indeed, both NUP98 and RAE1 have a nuclear and cytoplasmic pool. Strikingly, however, while NUP88 accumulated in the cytoplasm when overexpressed, cytoplasmic NUP98 and RAE1 levels remained unchanged, indicating that a stoichiometric imbalance between NUP88 and NUP98-RAE1 exists in the cytoplasm of Nup $88^{T}$ MEFs. Furthermore, NUP98 and RAE1 were predominantly lost from cytoplasmic fractions in $\mathrm{Nup} 98^{+/-} \mathrm{Rae}^{+/-} \mathrm{MEFs}$, without a concomitant loss of cytoplasmic NUP88 (Figure 4F), again creating a stoichiometric imbalance between NUP88 and NUP98RAE1. Together, these results suggest that in the $G_{2}$ phase, inhibition of cytoplasmic APC/C $\mathrm{C}^{\mathrm{CDH}}$ occurs via a cytoplasmic pool of NUP98-RAE1 not associated with NPCs and that soluble NUP88 competes with $\mathrm{APC} / \mathrm{C}^{\mathrm{CDH} 1}$ for binding of NUP98-RAE1. Moreover, these data indicate that NPCs are privileged sites that limit the bioavailability of soluble NUP88 and NUP98-RAE1 by first satisfying their structural requirements in order to maintain normal nucleocytoplasmic transport.

To directly test whether NUP88 sequesters NUP98-RAE1 away from $\mathrm{APC} / \mathrm{C}^{\mathrm{CDH} 1}$ in the cytoplasm, we first immunoprecipitated HA-Nup88 in cytoplasmic fractions of Nup88 $8^{T}$ MEFs and indeed discovered the existence of NUP88-NUP98-RAE1 complexes (Figure 4G). Next, we sought to compare the abundance of cytoplasmic NUP88-NUP98-RAE1 complexes between Nup88 ${ }^{T}$ and control MEFs. By immunoprecipitating endogenous NUP88 in cytoplasmic extracts and comparing coprecipitation of NUP98 and RAE1, we discovered that Nup $88^{T}$ MEFs had more cytoplasmic NUP88-NUP98-RAE1 complexes than did TA MEFs (Figure 4H). Together, we conclude that NUP88 sequesters NUP98-RAE1 away from $\mathrm{APC} / \mathrm{C}^{\mathrm{CDH} 1}$ when overexpressed and that this occurs in the cytoplasm, away from NPCs.

On the basis of these results, we predicted that cytoplasmic NUP88 serves as a reservoir for soluble NUP98-RAE1 complexes. If true, then reducing NUP88 protein levels in Nup98 $8^{+/-} \mathrm{Rael}^{+/-}$ MEFs would liberate sequestered NUP98-RAE1 to restore premi- 
A
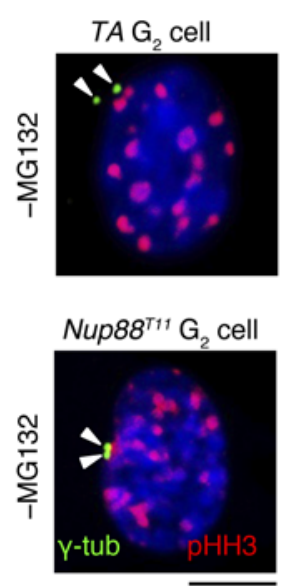

D

$85^{\circ}<$ Normal geometry $<95^{\circ}$

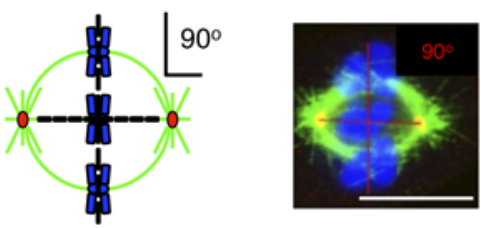

$85^{\circ}>$ Abnormal geometry $>95^{\circ}$
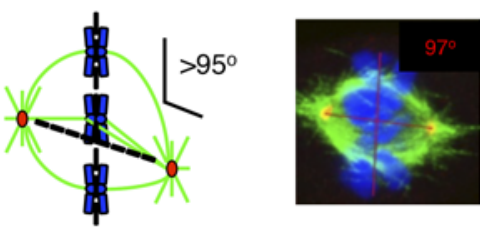

B

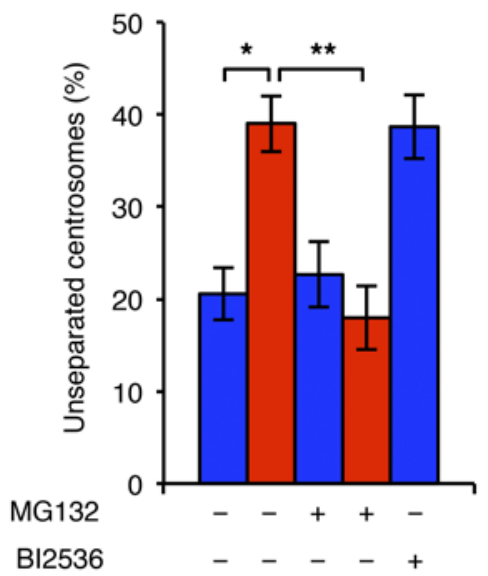

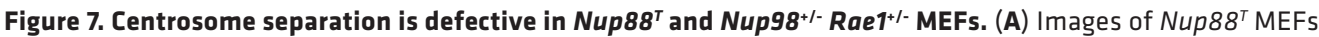
immunostained for $\gamma$-tubulin and p-HH3 (Ser10). (B) Quantification of the incidence of $\mathrm{G}_{2}$-phase cells of the indicated genotypes with unseparated centrosomes treated with or without BI2536. (C) Same as in B. (D) Schematic representation of spindle geometric measurements and representative images of metaphases immunostained for $\alpha$-tubulin and $\gamma$-tubulin with the indicated spindle angle. (E) Quantification of spindle geometric abnormalities for MEFs of the indicated genotypes. (F) Same as in $\mathbf{E}$. DNA in $\mathbf{A}$ and $\mathbf{D}$ was visualized with Hoechst. Quantifications in $\mathbf{B}, \mathbf{C}, \mathbf{E}$, and $\mathbf{F}$ were performed on 3 independent lines per genotype (20 cells/line). Data represent the mean \pm SEM. Statistical significance in B and $\mathbf{C}$ was determined using a 1-way ANOVA, followed by Tukey's multiple comparisons test. Statistical significance in $\mathbf{E}$ and $\mathbf{F}$ was determined using a 2-tailed, unpaired $t$ test. ${ }^{*} P<0.05$ and ${ }^{* *} P<0.01$. Scale bars: $10 \mu \mathrm{m}$. Nup88 $8^{T}$ indicates the combined transgenic lines 11 and 13 .

totic $\mathrm{APC} / \mathrm{C}^{\mathrm{CDH} 1}$ inhibition and ameliorate the mitotic abnormalities of Nup98-Rae1-haploinsufficient MEFs. To test this theory, we generated $\mathrm{Nup} 88^{+-}$mice using TALEN-mediated gene inactivation (Supplemental Figure 7) and crossed them onto a Nup98+/Rae1 ${ }^{+/-}$genetic background to obtain Nup $88^{+/-} \mathrm{Nup} 98^{+/-} \mathrm{Rael}^{1^{+-}}$ triple-mutant MEFs. We found that chromosome segregation errors were reduced from $37 \%$ in $\mathrm{Nup98^{+/- }} \mathrm{Rae}^{1^{+-}}$MEFs to $18 \%$ in triple-mutant MEFs and that the mitotic checkpoint was fully restored in triple-mutant MEFs (Figure 4, I and J). Consistently,

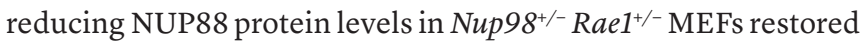
$\mathrm{G}_{2}$ - and $\mathrm{M}$-phase securin protein levels (Figure $4, \mathrm{~K}$ and L). Collectively, these data suggest that cytoplasmic NUP98-RAE1 normally inhibits APC/C ${ }^{C D H 1}$ but is sequestered away by NUP88 when overexpressed, thereby promoting the ubiquitin ligase activity of APC $/ \mathrm{C}^{\mathrm{CDH}}$.

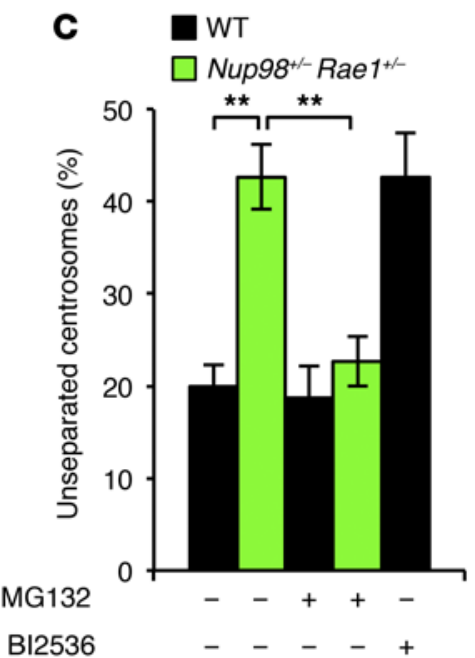

$\mathbf{F}$

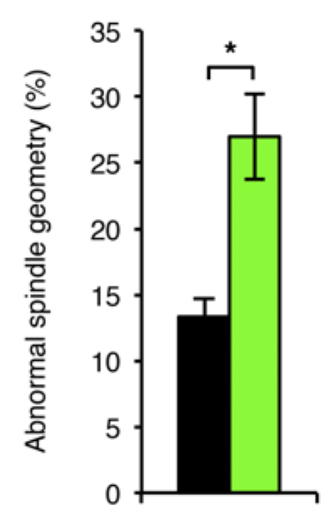

Nup98-Rae1 haploinsufficiency reveals premitotic $A P C / C^{\mathrm{CDH} 1}$ substrate selectivity. Next, we asked whether securin deficiency could explain the mitotic phenotypes of $N u p 88^{T}$ and Nup98 ${ }^{+/-} \mathrm{Rael}^{+/-}$MEFs. We found that securin-KO MEFs were karyotypically stable, had normal chromosome missegregation rates, and exhibited normal mitotic checkpoint activity (Supplemental Figure 8), suggesting that APC/ $\mathrm{C}^{\mathrm{CDH} 1}$ substrates other than securin were unstable during the $\mathrm{G}_{2}$ phase in Nup $88^{T}$ and $\mathrm{Nup} 8^{+/-} \mathrm{Rael}^{+/-}$MEFs. Western blot analysis of $\mathrm{G}_{2}$ MEFs demonstrated that aurora A, aurora B, CDC20, cyclin A2, and cyclin B1 protein levels were completely normal in $\mathrm{G}_{2}$-phase Nup98 $8^{+-} \mathrm{Rael}^{+/-} \mathrm{MEFs}$ (Figure $5 \mathrm{~A}$ ). However, levels of the tumor suppressor and mitotic kinase PLK1 were consistently lower in $\mathrm{Nup} 98^{+/-} \mathrm{Rael}^{+/-} \mathrm{MEFs}$ than the levels detected in control MEFs in both the $\mathrm{G}_{2}$ and mitosis phases (Figure 5, A and B). Although the observed substrate selectivity is striking and counterintuitive, $\mathrm{APC} / \mathrm{C}^{\mathrm{CDH} 1}$ has been shown to selectively target individual substrates, including PLK1, during interphase under various experimental conditions (23). Furthermore, we found that centrosomal PLK1 was reduced in $\mathrm{G}_{2}$-phase Nup $88^{T}$ and Nup $98^{+/-} \mathrm{Rael}^{+/-} \mathrm{MEFs}$, as indicated by the intensity of phosphorylated PLK1 (Thr210) (p-PLK1 [Thr210]) immunostaining (Figure 5, C-F). Importantly, these levels were fully restored upon proteasome inhibition. Finally, PLK1, like securin, was restored to WT levels in $\mathrm{G}_{2}$-phase Nup $88^{+/-} \mathrm{Nup98^{+/- }} \mathrm{Rae}^{1^{+-}}$MEFs (Figure 5, G

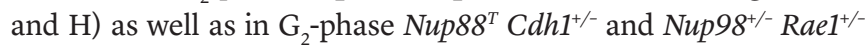
$C d h 1^{+-}$MEFs (Figure 5, I and J). Together, these data indicate that overexpression of NUP88 or low levels of NUP98-RAE1 license the premitotic degradation of select $\mathrm{APC} / \mathrm{C}^{\mathrm{CDH} 1}$ substrates, namely securin and PLK1.

Disruption of the NUP88-NUP98-RAE1 axis induces W-CIN through PLK1 loss. PLK1 is an essential serine/threonine kinase with several functions (24). To determine whether PLK1 insufficiency 
might account for the chromosome segregation defects observed in Nup $88^{T}$ and $N u p 98^{+/-} \mathrm{Rae}^{+/-}$MEFs, we depleted PLK1 from H2B-mRFP-positive WT MEFs using two shRNAs (Supplemental Figure 9A) and followed them via time-lapse microscopy. Indeed, PLK1 knockdown resulted in chromosome segregation errors characterized by high rates of lagging chromosomes and chromatin bridges (Figure 6A). The same missegregation abnormalities were observed when WT MEFs were grown in the presence of the PLK1 inhibitor BI2536 (Figure 6A), highlighting the role of the kinase activity of PLK1 in mediating proper chromosome segregation. We then investigated the extent to which PLK1 is the key APC/C $\mathrm{C}^{\mathrm{CDH} 1}$ substrate responsible for the observed mitotic defects by overexpressing PLK1 in Nup88 ${ }^{T}$ MEFs (Supplemental Figure 9B) and assessing chromosome missegregation rates. As shown in Figure 6B, PLK1 overexpression restored the chromosome missegregation phenotype of $N u p 88^{T}$ MEFs back to that seen at control levels. Collectively, these data indicate that precocious $\mathrm{APC} / \mathrm{C}^{\mathrm{CDH1}}$-mediated degradation of PLK1 is the key substrate responsible for induction of the chromosome missegregation observed in MEFs with perturbations of the NUP88-NUP98-RAE1 axis.

Next, we sought to identify how PLK1 insufficiency causes lagging chromosomes and chromatin bridges. Incomplete centrosome separation has recently been linked to merotelic attachment, the microtubule-kinetochore attachment defect that yields lagging chromosomes (25). Importantly, PLK1 is part of a signaling cascade that regulates centrosome separation (26). To determine whether centrosome separation is perturbed in $N u p 88^{T}$ and Nup98 $8^{+/-} \mathrm{Rae1}^{+/-}$MEFs, we immunostained MEFs for $\gamma$-tubulin and measured the amount of separation between sister centrosomes in the $G_{2}$ phase (Figure 7A and ref. 27). We found that incomplete centrosome separation was markedly increased in both $N u p 88^{T}$ and Nup98 $8^{+-} \mathrm{Rae1}^{+/-}$MEFs and that this exact centrosome separation defect was observed in control MEFs treated with BI2536 (Figure 7, $\mathrm{B}$ and $\mathrm{C})$. Importantly, normal centrosome separation was restored in $\mathrm{Nup} 88^{\mathrm{T}}$ and $\mathrm{Nup} 98^{+/-} \mathrm{Rae}^{+/-}$MEFs upon addition of MG132 (Figure 7, B and C), consistent with the concurrent restoration of total PLK1 and activated p-PLK1 (Thr210) protein levels (Figure 5, A-F).

Cells with impaired centrosome separation are prone to forming asymmetrical spindles that promote merotely and chromosome lagging (26). Consistently, spindle geometry defects were more frequent in Nup $88^{T}$ and $\mathrm{Nup} 98^{+/-} \mathrm{Rae1}^{+/-}$MEFs than in control MEFs (Figure 7, D-F). Notably, levels of aurora B and p-KNL1 were unchanged in Nup $88^{T}$ and Nup9 $8^{+/-} \mathrm{Rae}^{1^{+-}} \mathrm{MEFs}$, indicating that defects in the merotelic attachment error correction did not contribute to chromosome lagging (Supplemental Figure 10). Taken together, these data suggest that perturbation of the NUP88-NUP98-RAE1 axis promotes precocious APC/C $\mathrm{C}^{\mathrm{CDH} 1}$-mediated degradation of PLK1 in the $G_{2}$ phase, which in turn drives chromosome lagging by disrupting proper centrosome separation and preventing the establishment of spindle biorientation.

In contrast with lagging chromosomes, several molecular aberrations can cause chromatin bridges, including incomplete inner centromeric DNA decatenation, DNA replication errors, and impaired cleavage of the centromeric cohesin complex by separase. Topoisomerase II $\alpha$, which removes DNA catenation links between sister chromatids, was properly expressed and localized to inner centromeres in both $\mathrm{Nup} 88^{T}$ and $\mathrm{Nup} 98^{+/-} \mathrm{Rae1}^{+/-} \mathrm{MEFs}$ (Supplemental Figure 11, A-C), suggesting that bridges were not likely due to centromeric DNA entanglement. The same was true for DNA replication errors, as Nup $88^{T}, \mathrm{Nup98^{+/- }} \mathrm{Rael}^{+/-}$, and control MEFs showed similar rates of DNA synthesis and replication errors (Supplemental Figure 11D).

To investigate whether defective separase activity might

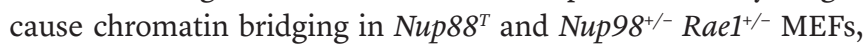
we used a biosensor that allows for real-time monitoring of separase-mediated cleavage of the cohesin subunit SCC1 (Figure $8 \mathrm{~A}$ and refs. 27, 28). We discovered that $\mathrm{Nup} 88^{\mathrm{T}}$ and $\mathrm{Nup} 98^{+/-}$ $\mathrm{Rae1}^{+/-}$MEFs with chromatin bridges inefficiently cleaved the biosensor, indicating reduced separase activity (Figure 8, B-E). PLK1 has been shown to promote separase activity by counteracting cyclin B1/CDK1-mediated inhibition of separase through phosphorylation of CDC6 (29), raising the possibility that premature PLK1 degradation in the context of NUP88 overexpression or Nup98-Rae1 haploinsufficiency induces chromatin bridge formation by promoting the inhibitory cyclin B1/CDK1-mediated hyperphosphorylation of separase. Consistently, WT MEFs subjected to shRNA-mediated knockdown of PLK1 exhibited reduced separase activity, particularly when chromatin bridges were present (Figure $8, \mathrm{~F}$ and $\mathrm{G}$ ). Thus, our data demonstrate that deregulation of the NUP88-NUP98-RAE1 axis drives premitotic APC/C ${ }^{\mathrm{CDH} 1}$-mediated degradation of PLK1, thereby blocking separase activity and promoting chromatin bridging in anaphase.

NUP88 overexpression promotes aneuploidy and PLK1 degradation in vivo. Genomic instability has been proposed to be an enabling characteristic of cancer (30). That is, CIN is thought to accelerate the acquisition of mutant genotypes during the early stages of transformation and subsequently confer a selective advantage. Consistently, aneuploidy has been shown to increase the incidence of cancer through tumor-suppressor loss of heterozygosity (31). In line with this, our data also suggest that NUP88 overexpression stimulates tumor initiation in mice. Moreover, our data indicate that NUP88 sequesters NUP98-RAE1 away from $\mathrm{APC} / \mathrm{C}^{\mathrm{CDH} 1}$ when overexpressed, thus establishing a CIN phenotype through the untimely degradation of PLK1. To determine whether this mechanism is relevant in vivo, we assessed aneuploidy rates and PLK1 levels in nontransformed lung tissue from 5-month-old $N u p 88^{T}$ mice. We reasoned that if the acquisition of a CIN phenotype is tumor initiating, then aneuploidy should be observed in lung tissue before overt tumors form. Indeed, lung tissues from $N u p 88^{T}$ mice were significantly more aneuploid than were control lung tissues as assessed by FISH and karyotypic analysis (Figure 9, A and B, and Supplemental Figure 12). Moreover, both PLK1 and securin protein levels were lower in $N u p 88^{T}$ lungs compared with the levels detected in TA controls (Figure 9, C and D). Together, these results are consistent with the idea that high levels of NUP88 promote aneuploidy through the untimely APC/C $\mathrm{C}^{\mathrm{CDH} 1}$-mediated degradation of PLK1 and securin in vivo in tumor-prone tissues.

\section{Discussion}

NUP88 is frequently elevated in human cancers, yet whether and how NUP88 overexpression drives tumorigenesis is unknown. Using transgenic mice, we found that NUP88 over- 
A
H2B

mCherry
hSCC1 (147-467 aa)

B

Nup88 normal anaphase
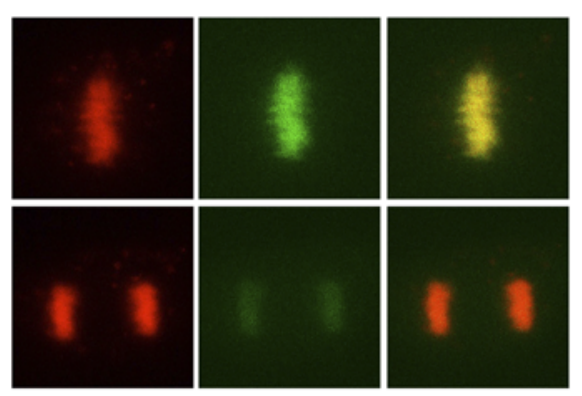

— TA normal anaphase

- Nup88 $8^{\top}$ normal anaphase

- Nup $88^{T}$ chromatin bridge

D
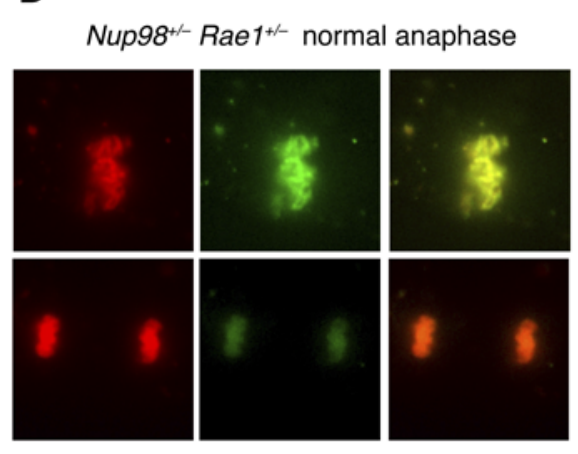

- WT normal anaphase

- Nup98 ${ }^{+-} \mathrm{Rae}^{+/-}$normal anaphase

- Nup98 ${ }^{+-}$Rae ${ }^{+/-}$chromatin bridge

$\mathbf{F}$

Control normal anaphase
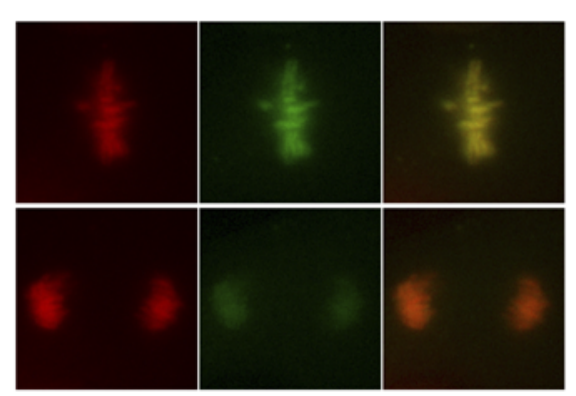

PIk1 shRNA chromatin bridge

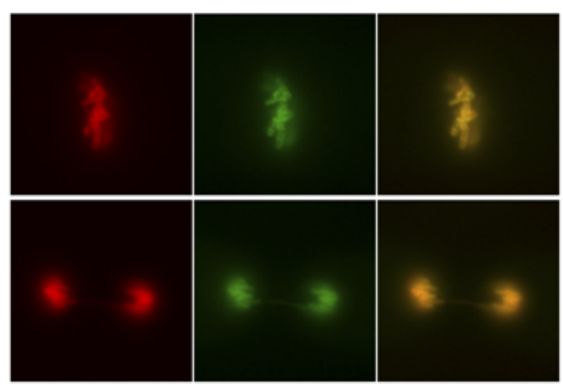

- Control normal anaphase

- Plk1 shRNA normal anaphase

- PIk1 shRNA chromatin bridge

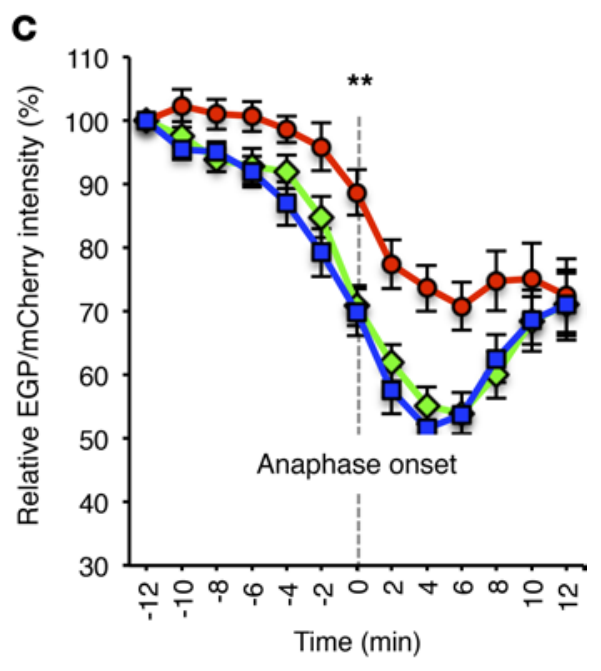

E
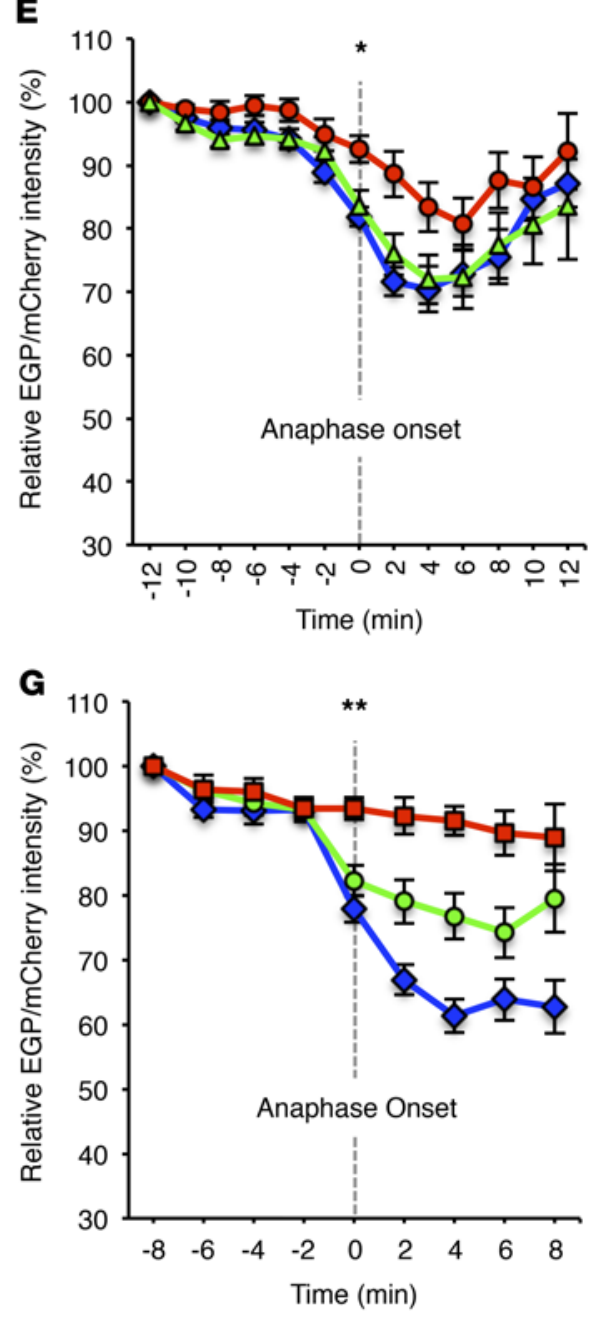
Figure 8. Perturbation of the NUP88-NUP98-RAE1 axis blocks separase activity via PLK1 insufficiency. (A) Schematic representation of the separase biosensor used for live-cell-imaging experiments. (B) Representative images of Nup88 MEFs expressing the separase biosensor before and after anaphase onset. (C) Quantification of biosensor cleavage for cells in B as they progressed through mitosis. (D) Representative images of Nup98 ${ }^{+/-}$Rae $^{+/-}$ MEFs expressing the separase biosensor before and after anaphase onset. (E) Quantification of biosensor cleavage for cells in $\mathbf{D}$ as they progressed through mitosis. (F) Representative images of WT MEFs transduced with Plk1 shRNA no. 1 expressing the separase biosensor before and after anaphase onset. (G) Quantification of biosensor cleavage for cells in $\mathbf{F}$ as they progressed through mitosis. Analyses in $\mathbf{C}$, $\mathbf{E}$, and $\mathbf{G}$ were performed on at least 10 cells per genotype from 2 independent lines. Data represent the mean \pm SEM. Statistical significance at anaphase onset $(T=0 \mathrm{~h})$ of mutant chromatin bridges versus control normal anaphases in $\mathbf{C}, \mathbf{E}$, and $\mathbf{G}$ was determined using a 1-way ANOVA, followed by Tukey's multiple comparisons test. ${ }^{*} P<0.05$ and ${ }^{*} P<0.01$. Scale bars: $10 \mu \mathrm{m}$. Nup $88^{T}$ indicates the combined transgenic lines 11 and 13 .

expression causes cancer, including tumor types for which it serves as a prognostic biomarker. We also identified NUP88 and the NUP98-RAE1 complex as core components of a regulatory axis that ensures accurate chromosome segregation by controlling premitotic $\mathrm{APC} / \mathrm{C}^{\mathrm{CDH} 1}$ activity against select substrates. NPCs have been reported to catalyze the formation of a MAD1-dependent APC/C ${ }^{C D C 20}$ inhibitor prior to mitosis, which is important for regulating proper mitotic timing and maintaining genomic integrity (6). Here, we show that individual NPC components and subcomplexes ensure accurate chromosome segregation and proper centrosome separation by regulating premitotic $\mathrm{APC} / \mathrm{C}^{\mathrm{CDH} 1}$ activity. Together, these studies identify what we believe to be a novel biological principle that the nuclear transport machinery safeguards against merotely induced genomic instability through two distinct yet complementary mechanisms: first, the soluble NUP88-NUP98-RAE1 axis ensures proper execution of premitotic centrosome separation; and second, NPC-catalyzed MCCs prolong the duration of mitosis so that the error correction network has sufficient time to destabilize faulty merotelic attachments.

Several lines of evidence presented here demonstrate that NUP98-RAE1-APC/C $\mathrm{C}^{\mathrm{CDH} 1}$ complexes exist prior to mitosis; that premitotic $\mathrm{APC} / \mathrm{C}^{\mathrm{CDH}}$ is normally inhibited by the NUP98-RAE1 complex; that NUP88 perturbs the inhibitory effect of NUP98RAE1 by sequestering it away from APC/ $\mathrm{C}^{\mathrm{CDH} 1}$; that PLK1 is the key APC/C $\mathrm{C}^{\mathrm{CDH} 1}$ substrate affected by NUP88 overexpression; and that low premitotic PLK1 levels are responsible for the chromosome missegregation defects observed in Nup $88^{T}$ MEFs. First, there existed a pool of preformed APC/ $\mathrm{C}^{\mathrm{CDH1}}$ that was actively bound to the NUP98-RAE1 complex prior to mitosis (Figure 4A). Second, NUP98-RAE1 was determined to inhibit APC/ $\mathrm{C}^{\mathrm{CDH1}}$ activity, because Nup $98^{+-} \mathrm{Rae}^{+-} \mathrm{MEFs}$ were unable to fully accumulate securin or PLK1 prior to mitosis (Figures 3 and 5). Consistently, PLK1 levels were restored upon concomitant reduction of $\mathrm{CDH} 1$, and securin levels were restored upon treatment with MG132 and proTAME, but not apcin (Figure 5, I and J). Third, NUP88 overexpression mimicked the mitotic phenotypes of Nup98-Rae1 haploinsufficiency (Figure 2), suggesting that NUP88 perturbs the protective effects of the NUP98-RAE1 complex. Like Nup98+Rae ${ }^{+/}$MEFs, Nup $88^{T}$ MEFs were also unable to fully accumulate securin or PLK1 prior to mitosis, and these defects could be corrected using the same strategies of $\mathrm{CDH} 1$ reduction and treatment with MG132 and proTAME, but not with apcin (Figures 3 and 5). Fourth, NUP88 bound NUP98-RAE1 independently of APC/ $\mathrm{C}^{\mathrm{CDH} 1}$ (Figure 4B) and decreased the abundance of inhibitory NUP98RAE1-APC/ $\mathrm{C}^{\mathrm{CDH} 1}$ complexes when overexpressed (Figure $4 \mathrm{C}$ and Supplemental Figure 6). Moreover, reducing NUP88 in the context of Nup98-Rae1 haploinsufficiency was sufficient to restore APC/ $\mathrm{C}^{\mathrm{CDH1}}$ inhibition (Figure 4, I-L, and Figure 5, G and H), presumably by reestablishing the optimal stoichiometric ratio between NUP88-NUP98-RAE1 and NUP98-RAE1-APC/C ${ }^{\text {CDH1 }}$ complexes. Fifth, lentiviral-mediated PLK1 overexpression was sufficient to rescue the chromosome segregation defects in Nup $88^{T}$ MEFs (Figure 6B), strengthening the idea that premature PLK1 degradation is the key molecular event responsible for the CIN phenotype of cells with elevated NUP88. Sixth, low PLK1 protein levels reproduced the chromosome segregation defects observed in Nup $88^{T}$ and $\mathrm{Nup} 8^{+-} \mathrm{Rael}^{+/-}$MEFs (Figure 6A), further strengthening the idea that untimely PLK1 degradation is the key abnormality responsible for the CIN phenotype seen in these mutant cell lines. Indeed, reduced PLK1 levels have previously been linked to abnormal centrosome dynamics prior to NEBD as well as reduced separase activity $(26,29)$.

The data presented here implicate PLK1, rather than securin, as the critical $\mathrm{APC} / \mathrm{C}^{\mathrm{CDH}}$ target that NUP98-RAE1 is tasked to protect prior to mitosis. But why are not all $\mathrm{APC} / \mathrm{C}^{\mathrm{CDH} 1}$ substrates prematurely degraded in the settings of Nup98-Rae1 haploinsufficiency and NUP88 overexpression? Although the phenomenon of APC/C substrate selectivity is well established (8), it remains poorly understood. As it pertains to the current study, our model demonstrates that when NUP88 accumulates in the cytoplasm, it sequesters soluble NUP98-RAE1 away from the pool of APC/ $\mathrm{C}^{\mathrm{CDH1}}$ that is also located in the cytoplasm. This triggers the selective premature activation of the cytoplasmic pool of APC/ $\mathrm{C}^{\mathrm{CDH} 1}$. Because the majority of $\mathrm{APC} / \mathrm{C}^{\mathrm{CDH} 1}$ substrates are nuclear, they are protected by virtue of the subcellular partitioning imposed by the NE. However, securin and PLK1 are located to varying extents on both sides of the NE, and their cytoplasmic pools are subsequently prone to $\mathrm{APC} / \mathrm{C}^{\mathrm{CDH}}$-mediated degradation, leading to centrosome separation defects and chromosome missegregation.

While PLK1 is an established $\mathrm{APC} / \mathrm{C}^{\mathrm{CDH} 1}$ substrate, it is widely accepted that $\mathrm{APC} / \mathrm{C}^{\mathrm{CDC} 20}$, not $\mathrm{APC} / \mathrm{C}^{\mathrm{CDH} 1}$, degrades securin during metaphase to release separase and promote chromosome disjunction. However, the proteasome-mediated regulation of securin in the $G_{2}$ phase does not seem to conform to this dogma. Surprisingly, $\mathrm{G}_{2}$-phase securin levels in BUBR1-null MEFs are completely indistinguishable from those in WT MEFs (18). However, Nup98-Rae1 haploinsufficiency markedly reduced $\mathrm{G}_{2}$-phase securin levels, suggesting that NUP98-RAE1-mediated inhibition of $\mathrm{APC} / \mathrm{C}^{\mathrm{CDH}}$, rather than MCC-mediated inhibition of APC/ $\mathrm{C}^{\mathrm{CDC20}}$, is critical for the accumulation of securin prior to mitosis. These findings are consistent with those of earlier studies demonstrating that both $\mathrm{APC} / \mathrm{C}^{\mathrm{CDC} 20}$ and $\mathrm{APC} / \mathrm{C}^{\mathrm{CDH}}$ efficiently polyubiquitylate securin (32). 
A

Lung
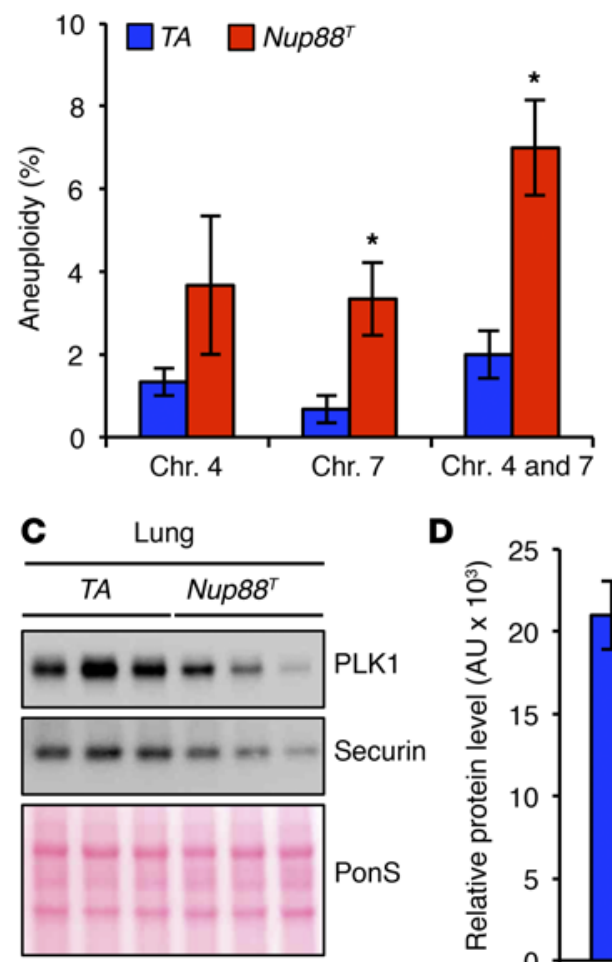

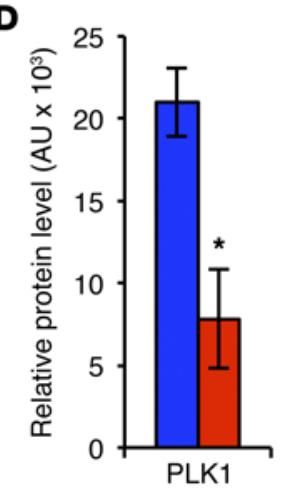

B Lung cell culture

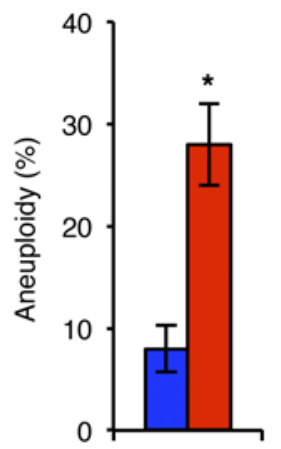

Figure 9. NUP88 overexpression promotes aneuploidy and PLK1 insufficiency in vivo. (A) Quantification of chromosome (Chr.) 4 and 7 copies by interphase FISH in lung tissue harvested from 5-month-old mice of the indicated genotypes. (B) Karyotype analysis of numerical chromosomal abnormalities in cell cultures established from minced lung tissue harvested from 5-monthold transgenic mice. (C) Western blot analysis of lung tissue harvested from 5-month-old mice of the indicated genotypes. (D) Quantification of PLK1 and securin protein levels from C. Analysis in $\mathbf{A}$ was performed on 3 independent lines per genotype (100 cells/line). Analysis in B was performed on 3 independent lines per genotype ( 25 spreads/line). Analysis in $\mathbf{D}$ was performed on 3 independent samples per genotype. Data represent the mean \pm SEM. Western blots are representative of 3 independent experiments. Statistical significance was determined in A, B, and D using a 2-tailed, unpaired $t$ test. ${ }^{*} P<0.05$. Nup $88^{T}$ indicates the combined transgenic lines 11 and 13.

overexpression, PLK1 and securin are selectively targeted for degradation. Therefore, the transformative potential of NUP88 overexpression is closely linked to securin and PLK1 loss. Because securin loss by itself does not lead to an overt cancer phenotype in mice (37), securin insufficiency does not seem to be a good candidate for driving tumorigenesis in $N u p 88^{T}$ mice. In contrast, PLK1 is a haploinsufficient tumor suppressor and is therefore a strong candidate for a key driver of transformation.

While NUP88 overexpression leads to degradation of the tumor suppressor PLK1, it is not yet known what

PLK1 has several mitotic functions (24) that make it an important regulator of chromosomal and genomic stability. However, the functions of PLK1 are not limited to mitosis. In fact, PLK1 kinase activity is indispensable for NEK2A-mediated centrosome separation in the late $G_{2}$ phase $(26,33)$. Cells with delayed centrosome separation are highly prone to chromosome lagging (25), the most common chromosome segregation error detected in human cancer cells. While not all mouse models of aneuploidy have a cancer predisposition, every mouse model with abnormal centrosome dynamics - cyclin B2 overexpression, USP44 KO, and NUP88 overexpression - is tumor prone $(27,34)$. Thus, abnormal centrosome dynamics may invoke a uniquely dangerous form of aneuploidy more capable of facilitating malignant transformation and/or tumor progression than other sources of CIN, highlighting the importance of premitotic protection of PLK1 by the NUP98-RAE1 complex.

Here, we provide direct evidence that NUP88 overexpression drives tumorigenesis. We show that NUP88 overexpression promotes chromosome missegregation via premature APC/ $\mathrm{C}^{\mathrm{CDH} 1}$-mediated PLK1 degradation. An earlier study established $\mathrm{APC} / \mathrm{C}^{\mathrm{CDH} 1}$ as a negative regulator of proliferation and tumor suppression (35). How can APC/C $\mathrm{C}^{\mathrm{CDH} 1}$ have such a paradoxical role in tumorigenesis? The key difference is that depletion of CDH1 results in a global reduction of $\mathrm{APC} / \mathrm{C}^{\mathrm{CDH} 1}$ activity and subsequent tumorigenesis, whereas NUP88 overexpression results in the selective $\mathrm{APC} / \mathrm{C}^{\mathrm{CDH} 1}$-mediated degradation of securin and PLK1, the latter of which is a haploinsufficient tumor suppressor (36). Because many APC/C $\mathrm{C}^{\mathrm{CDH} 1}$ substrates have transformative potential, the net effect of global degradation of these proteins is to suppress tumorigenesis. However, in the context of NUP88
PLK1-mediated cellular and/or molecular process(es) actually drive tumorigenesis. However, one attractive candidate is aneuploidy, which is a hallmark of nearly all cancers, and most mouse models with mutations in CIN genes predispose the animals to cancer. Nonetheless, cancer is not an obligatory outcome of aneuploidy (38). However, in the case of NUP88 overexpression, it is reasonable to suspect that one mechanism whereby elevated NUP88 drives tumorigenesis is through PLK1 destabilization and aneuploidization. First, PLK1 is a haploinsufficient tumor suppressor that ensures faithful chromosome segregation in mitosis. To this end, Plk1-heterozygous mice develop spontaneous tumors and have high levels of aneuploidy (36). Second, the exacerbation of colon cancer incidence in $A P C^{+/ M i n}$ mice that overexpress NUP88 is consistent with the notion that aneuploidy promotes tumorigenesis through tumor-suppressor loss of heterozygosity (31). Which of the many functions of PLK1 are important for tumor suppression and the precise role aneuploidy plays in PLK1-mediated tumor suppression will be important questions to investigate in the future.

\section{Methods}

Mouse strains. Mice used in these studies were housed in a pathogen-free barrier and were mixed on a $129 / \mathrm{Sv} \times \mathrm{C} 57 \mathrm{BL} / 6$ background. Nup $88^{T}$ mice were generated as previously described (15, 27). Briefly, WT Nup 88 cDNA with a $5^{\prime}$ HA tag was inserted into the EcoRI site of the pBS31'-SR $\alpha$ FLP-in vector (gift of Rick Bram, Mayo Clinic, Rochester, Minnesota, USA). The resultant plasmid was electroporated into KH2 ES cells (Origene Technologies) with pCAGGs-FLPe-puro to target HA-Nup88 to an FLP recognition site downstream of Col1a1. KH2 ES cells were injected into blastocysts, 
and chimeras from two independent clones achieved germline transmission (Nup88 $8^{T 11}$ and $\left.N u p 88^{T 13}\right)$. Nup $88^{T}$ mice were maintained on an M2-rtTA (TA) hemizygous background and continually administered $2 \mathrm{mg} / \mathrm{ml}$ dox in drinking water containing $5 \%$ sucrose after weaning. Male and female mice were used for experimentation. At 14 months of age, the mice were sacrificed and screened for overt tumors. Mice on the $A P C^{+/ M i n}$ genetic background (17) were administered dox water after weaning age for 60 or 90 days, sacrificed on day 90, and processed as described (27). Nup88-heterozygous mice were generated using TALEN-mediated gene targeting as previously described (39). The TALEN recognition sequences were 5'-GTTAGTTCAAGATGG-3' and 5'-CCCCATCGCCCAAGG-3', and the RVD sequences were $5^{\prime}$-NN-NG-NG-NI-NN-NG-NG-HDNI-NI-NN-NI-NG-NN-NN-3' and 5'-HD-HD-HD-HD-NI-NG-HDNN-HD-HD-HD-NI-NI-NN-NN-3'. Pronuclear injection of $100 \mathrm{ng} /$ $\mu$ TALEN mRNA yielded three founders: two had distinct 3-bp inframe deletions, and one had an 11-bp frame-shift deletion on one Nup88 allele (KO allele) and a 12-bp in-frame deletion on the second allele. We bred the KO founder with a WT C57BL/6 mouse to isolate and maintain the $\mathrm{KO}$ allele on a $\mathrm{Nup} 88^{+/-}$genetic background. To generate the $C d h 1-\mathrm{KO}$ allele, we obtained the $C d h 1$ gene-trap ES cell line (RRJ607) from BayGenomics and performed blastocyst injection to yield heterozygous carriers (40). The generation of $\mathrm{Nup98^{+/- }}$ and $\mathrm{Rael}^{+/-}$mice has been previously described $(41,42)$. Securin-KO mice were a gift of Pumin Zhang (Baylor College of Medicine, Houston, Texas, USA) (43).

Generation and culture of MEFs and primary lung cells. MEF generation and culturing were performed as previously described (42). Primary MEFs were frozen at passage 2 (P2) or P3 and used for experimentation between $\mathrm{P} 4$ and P6. At least three independently generated MEF lines per genotype were used. To induce HA-Nup 88 expression, $2 \mu \mathrm{g} / \mathrm{ml}$ dox was added to the culture medium 48 hours before analysis. Primary lung cultures were established by culturing minced lung tissue in fully supplemented DMEM containing $10 \%$ FCS and $2 \mu \mathrm{g} / \mathrm{ml}$ dox.

Karyotype analysis and interphase FISH. Chromosome counts of metaphase spreads were performed as previously described (42). P5 transgenic MEFs were cultured in dox media for 48 hours. Colcemid was added to the culture for 4 hours, and the cells were swelled with $0.075 \mathrm{M} \mathrm{KCl}$, fixed, and stained with Giemsa. Splenocytes were harvested from 5-month-old transgenic mice that had received dox since weaning age, and the splenocytes were processed identically. Fifty cells were analyzed per sample. Interphase FISH of chromosomes 4 and 7 was performed as described previously (31). Briefly, lung tissue was minced using a dissociator (gentleMACS; Miltenyi Biotec) and enzymatically digested with liberase (Roche). Single-cell suspensions were then hybridized with probes corresponding to chromosomes $4 \mathrm{Eq} 2$ and 7qA1. One hundred cells were analyzed per sample.

Live-cell imaging. Chromosome segregation analysis, mitotic progression analysis, and nocodazole challenge assays were performed on MEFs stably expressing H2B-mRFP, as previously described (27). All chromosome segregation error analyses are representative of three independent experiments. The separase biosensor experiment was performed and quantified as previously described (27). For quantitative analysis of securin-EYFP accumulation, primary MEFs were cotransduced with lentiviruses expressing securin-EYFP and H2B-mRFP and followed at 7-minute intervals until the completion of mitosis. NEBD was assigned the 0-minute time point, and all securin-EYFP intensity values were normalized against it. The securin cDNA fragment from pEYFP-N1-securin (provided by Jonathan Pines, Gurdon Institute, Cambridge, England, United Kingdom) was cloned into the lentiviral expression vector TRIPz3. Securin-EYFP intensity measurements were obtained using ImageJ software (NIH) as described previously (12).

Western blot analysis, cellular synchronization, subcellular fractionation, and co-IP. Western blot analyses were performed as described previously (27). Mitotic cell lysates were prepared by adding nocodazole $(100 \mathrm{ng} / \mathrm{ml})$ to culture for 4 to 5 hours, followed by mechanical agitation. $G_{2}$ synchronization was performed by serum starving superconfluent MEFs in 0.1\% FBS dox media for 14 hours $(T=0 \mathrm{~h})$. Cells were then trypsinized and released into $20 \%$ FBS dox media. After 18 hours, RO-3306 was added, and 4 hours later, MG132 was added. One hour after addition of MG132, the lysates were prepared $\left(T=23 \mathrm{~h}\right.$ ). HeLa cells were synchronized in $G_{2}$ as previously described (21). Subcellular fractionation was performed using the Subcellular Protein Fractionation Kit (Thermo Scientific) according to the manufacturer's instructions. Co-IP was performed as previously described (44). Primary Abs used for Western blotting were: rat anti-HA-peroxidase (clone 3F10, 12013819001, 1:1,000; Roche); mouse anti-NUP88 (clone H-7, sc-365868, 1:20,000; Santa Cruz Biotechnology Inc.); mouse anti-CDC27 (610455, 1:1,000; BD Transduction Laboratories); mouse anti-RanBP2 (clone D-4, sc-74518, 1:1,000; Santa Cruz Biotechnology Inc.); mouse anti- $\beta$ actin (clone AC-15, 061M4808, 1:40,000; Sigma-Aldrich); mouse anti-securin (DCS-280, ab3305, 1:1,000; Abcam); rabbit anti-cyclin B1 (4138S, 1:1,000; Cell Signaling Technology); mouse anti-BUBR1 (612503, 1:1,000; BD Transduction Laboratories); goat anti-CDC16/ APC6 (clone K-16, sc-6395, 1:1,000; Santa Cruz Biotechnology Inc.); mouse anti-CDH1 (FZR1) (DCS-266, ab3242, 1:1,000; Abcam); mouse anti-CDC20 (p55) (clone H-7, sc-5296, 1:1,000; Santa Cruz Biotechnology Inc.); mouse anti-PLK1 (clone F-8, sc-17783, 1:1,000; Santa Cruz Biotechnology, Inc.); rabbit anti-p-HH3 (Ser10) (06-570, 1:40,000; EMD Millipore); rabbit anti-RAE1 (1:20,000, described in ref. 45); rabbit anti-NUP98 (1:10,000; described in ref. 44); rabbit anti-CRM1 (1:10,000; provided by Maarten Fornerod, Erasmus University, Rotterdam, Netherlands); rabbit anti-cyclin A2 antiserum (1:80,000; in-house); affinity-purified rabbit anti-NUP214 antiserum (1:1,000; in-house); mouse anti-AIM-1/aurora B (611083, 1:2,000; BD Transduction Laboratories); mouse anti-aurora A (ab13824, 1:1,000; Abcam); rabbit anti-topo II $\alpha$ (1:1,000; Topogen); mouse anti- $\alpha$-tubulin (clone DM1A, T-9026, 1:20,000; SigmaAldrich); and rabbit anti-H3 (1:10,000; gift of Zhiguo Zhang, Mayo Clinic, Rochester, Minnesota, USA). Ponceau S staining served as a loading control for tissue blots. All Western blots are representative of at least three independent experiments.

Indirect immunofluorescence and confocal microscopy. Immunofluorescence was performed as previously described (27). For nuclear rim staining, cells were fixed in $1 \mathrm{X} \mathrm{PBS} / 3 \%$ paraformaldehyde (PFA) for 5 minutes at room temperature (RT), permeabilized in $1 \mathrm{X}$ PBS/0.2\% Triton X-100 for 15 minutes, and blocked in $1 \mathrm{X}$ PBS/5\% BSA for 60 minutes at RT. For whole-cell fixations, cells were fixed in $1 \mathrm{X}$ PBS $/ 3 \%$ PFA for 15 minutes at RT before permeabilization and blocking. Spindle geometry, centrosomal p-PLK (Thr210) intensity, 
and centrosome separation (27); aurora B and pKNL intensity (46); and topoisomerase II $\alpha$ localization (47) studies were performed as previously described. All confocal microscopic images are representative of at least three independent experiments. Primary Abs used for immunostaining were: mouse anti- $\gamma$-tubulin (T6557/ clone GTU-88, 1:300; Sigma-Aldrich); rabbit anti- $\gamma$-tubulin (T5192, 1:500; Sigma-Aldrich); mouse anti- $\alpha$-tubulin (T9026/clone DM1A, 1:1,000; Sigma-Aldrich); rabbit anti-HA (3724, 1:250; Cell Signaling Technology); mouse anti-p-PLK (Thr210) (ab39068/clone 2A3, 1:300; Abcam); rabbit anti-p-HH3 (Ser10) (06-570, 1:10,000; EMD Millipore); mouse anti-NUP88 (clone H-7, sc-365868, 1:250; Santa Cruz Biotechnology Inc.); mouse anti-AIM-1/aurora B (611083, 1:3,000; BD Transduction Laboratories); rabbit anti-p-KNL (Ser24) (1:2,000; provided by Ian Cheeseman, Whitehead Institute for Biomedical Research, Cambridge, Massachusetts, USA); human anti-centromeres (15-235-0001, 1:100; Antibodies Inc.); rabbit anti-topo II $\alpha$ (1:300; Topogen); mouse anti-CDC27 (610455, 1:100; BD Transduction Laboratories); goat anti-CDC16 (APC6) (clone K-16, sc-6395, 1:100; Santa Cruz Biotechnology Inc.); goat anti-p53 (clone FL-393, sc-6243-G, 1:100; Santa Cruz Biotechnology Inc.); p-histone H2Ax (Ser139) (JBW301, 05-636, 1:100; EMD Millipore); mouse anti-NPM1 (325200, 1:100; Invitrogen); and rabbit anticyclin B1 (4138S, 1:200; Cell Signaling Technology). CDH1 subcellular localization was performed by transfecting WT MEFs with a CDH1-GFP expression construct (22) (gift of A. Bonni, Washington University, St. Louis, Missouri, USA) 24 hours before fixation using the Amaxa MEF2 Nucleofector Kit and device (Lonza) according to the manufacturer's protocol.

DNA fiber assay. DNA fiber assays were performed as previously described (48).

Lentiviral transduction. The nontargeting shRNA TRC2 negative control (SCH2O2) and Plk1 shRNA TRC2 clones NM-011121.31338s21c1, NM-011121.3-1416s21c1, NM-011121.3-1711s21c1, NM-011121.3-1944s21c1, and NM-011121.3-567s21c1 were purchased from Sigma-Aldrich, and lentiviruses were prepared according to the manufacturer's instructions. Cells were infected with Plk1 shRNA or nontargeting shRNA for 48 hours, selected with puromycin $(2 \mu \mathrm{g} / \mathrm{ml})$ for 48 hours, and analyzed the next day. PLK1 overexpression was induced as previously reported (49) (the PLK1 lentiviral construct was a gift of Paul Hwang, NIH, Bethesda, Maryland, USA).

Nuclear transport assays. Nuclear transport assays were performed as described previously (50).

Statistics. GraphPad Prism software was used for all statistical analyses. Statistical significance for comparisons was determined by 2-tailed, unpaired $t$ test; 1-way ANOVA, followed by Tukey's multiple comparisons test; 2-tailed Fisher's exact test; and 1-tailed Fisher's exact test with Bonferroni's correction. A $P$ value of less than 0.05 was considered statistically significant. Sample sizes for spontaneous tumor studies and $A P C^{+/ M i n}$ studies were chosen on the basis of power analysis and previously published studies in which differences were observed. No samples were excluded. The experiments were not randomized, and the investigators were not blinded.

Study approval. All protocols were reviewed and approved by the IACUC of Mayo Clinic (protocol A2314).

\section{Author contributions}

JMV conceived the project. RMN and JMV designed the research studies. RMN, KBJ, and $\mathrm{XC}$ conducted the experiments and acquired the data. RMN, JMV, KBJ, and XC analyzed the data. $\mathrm{RMN}$ and JMV wrote the manuscript.

\section{Acknowledgments}

We thank Wei Zhou and Ming Li of Mayo Clinic's Gene Knockout Mouse Core Facility; Chris Ward and Jason Bakeberg for help in designing and cloning TALENs; Darren Baker, Hyunja Nam, Robbyn Weaver, and Khaled Aziz for critical reading of the manuscript; Arun Kanakkanthara for assistance with the DNA fiber assay; and Pumin Zhang, Zhiguo Zhang, Paul Hwang, Jonathan Pines, Ian Cheeseman, Maarten Fornerod, Rick Bram, and Azad Bonni for sharing helpful reagents. This work was supported by grants from the NIH (F30 CA189339-01 and T32 GM65841, to R.M. Naylor, and R01 CA096985 and R01 CA126828, to J.M. van Deursen).

Address correspondence to: Jan M. van Deursen, Mayo Clinic, 200 First St. SW, Rochester, Minnesota 55905, USA. Phone: 507.284.2524; E-mail: vandeursen.jan@mayo.edu.
1. Xu S, Powers MA. Nuclear pore proteins and cancer. Semin Cell Dev Biol. 2009;20(5):620-630.

2. Gould VE, Orucevic A, Zentgraf H, Gattuso P, Martinez N, Alonso A. Nup88 (karyoporin) in human malignant neoplasms and dysplasias: correlations of immunostaining of tissue sections, cytologic smears, and immunoblot analysis. Hum Pathol. 2002;33(5):536-544.

3. Emterling A, et al. Clinicopathological significance of Nup88 expression in patients with colorectal cancer. Oncology. 2003;64(4):361-369.

4. Hashizume C, Nakano H, Yoshida K, Wong RW. Characterization of the role of the tumor marker Nup88 in mitosis. Mol Cancer. 2010;9:119.

5. Wozniak R, Burke B, Doye V. Nuclear transport and the mitotic apparatus: an evolving relationship. Cell Mol Life Sci. 2010;67(13):2215-2230.

6. Rodriguez-Bravo V, et al. Nuclear pores protect genome integrity by assembling a premitotic and Mad1-dependent anaphase inhibitor. Cell. 2014;156(5):1017-1031

7. Musacchio A, Salmon ED. The spindle-assembly checkpoint in space and time. Nat Rev Mol Cell Biol. 2007;8(5):379-393.

8. Pines J. Cubism and the cell cycle: the many faces of the APC/C. Nat Rev Mol Cell Biol. 2011;12(7):427-438.

9. Sivakumar S, Gorbsky GJ. Spatiotemporal regulation of the anaphase-promoting complex in mitosis. Nat Rev Mol Cell Biol. 2015;16(2):82-94.

10. Listovsky T, Sale JE. Sequestration of CDH1 by MAD2L2 prevents premature APC/C activation prior to anaphase onset. JCell Biol. 2013;203(1):87-100.

11. Jeganathan KB, Baker DJ, van Deursen JM. Securin associates with APCCdh1 in prometaphase but its destruction is delayed by Rae 1 and Nup98 until the metaphase/anaphase transition.
Cell Cycle. 2006;5(4):366-370.

12. Jeganathan KB, Malureanu L, van Deursen JM. The Rae1-Nup98 complex prevents aneuploidy by inhibiting securin degradation. Nature. 2005;438(7070):1036-1039.

13. Salsi V, et al. NUP98 fusion oncoproteins promote aneuploidy by attenuating the mitotic spindle checkpoint. Cancer Res. 2014;74(4):1079-1090.

14. Lara-Gonzalez P, Westhorpe FG, Taylor SS. The spindle assembly checkpoint. Curr Biol. 2012;22(22):R966-R980.

15. Hochedlinger K, Yamada Y, Beard C, Jaenisch R. Ectopic expression of Oct-4 blocks progenitorcell differentiation and causes dysplasia in epithelial tissues. Cell. 2005;121(3):465-477.

16. Gould VE, Martinez N, Orucevic A, Schneider J, Alonso A. A novel, nuclear pore-associated, widely distributed molecule overexpressed in oncogenesis and development. Am J Pathol. 
2000;157(5):1605-1613.

17. Su LK, et al. Multiple intestinal neoplasia caused by a mutation in the murine homolog of the APC gene. Science. 1992;256(5057):668-670.

18. Malureanu LA, Jeganathan KB, Hamada M, Wasilewski L, Davenport J, van Deursen JM. BubR1 $\mathrm{N}$ terminus acts as a soluble inhibitor of cyclin B degradation by APC/C(Cdc20) in interphase. Dev Cell. 2009;16(1):118-131.

19. Zeng $X$, et al. Pharmacologic inhibition of the anaphase-promoting complex induces a spindle checkpoint-dependent mitotic arrest in the absence of spindle damage. Cancer Cell. 2010;18(4):382-395.

20. Sackton KL, et al. Synergistic blockade of mitotic exit by two chemical inhibitors of the APC/C. Nature. 2014;514(7524):646-649.

21. Vassilev LT, et al. Selective small-molecule inhibitor reveals critical mitotic functions of human CDK1. Proc Natl Acad Sci U S A. 2006;103(28):10660-10665.

22. Stegmüller J, Konishi Y, Huynh MA, Yuan Z, DiBacco S, Bonni A. Cell-intrinsic regulation of axonal morphogenesis by the Cdh1-APC target SnoN. Neuron. 2006;50(3):389-400.

23. Bassermann F, Frescas D, Guardavaccaro D, Busino L, Peschiaroli A, Pagano M. The Cdc14B-Cdh1-Plk1 axis controls the G2 DNA-damage-response checkpoint. Cell. 2008;134(2):256-267.

24. Petronczki M, Lenart P, Peters JM. Polo on the rise-from mitotic entry to cytokinesis with Plk1. Dev Cell. 2008;14(5):646-659.

25. Silkworth WT, Nardi IK, Paul R, Mogilner A, Cimini D. Timing of centrosome separation is important for accurate chromosome segregation. Mol Biol Cell. 2012;23(3):401-411.

26. Nam HJ, Naylor RM, van Deursen JM. Centrosome dynamics as a source of chromosomal instability. Trends Cell Biol. 2015;25(2):65-73.

27. Nam HJ, van Deursen JM. Cyclin B2 and p53 control proper timing of centrosome separation. Nat Cell Biol. 2014;16(6):538-549.

28. Shindo N, Kumada K, Hirota T. Separase sensor reveals dual roles for separase coordinating cohesin cleavage and cdk1 inhibition. Dev Cell. 2012;23(1):112-123.
29. Yim H, Erikson RL. Cell division cycle 6, a mitotic substrate of polo-like kinase 1 , regulates chromosomal segregation mediated by cyclin-dependent kinase 1 and separase. Proc Natl Acad Sci U S A. 2010;107(46):19742-19747.

30. Hanahan D, Weinberg RA. Hallmarks of cancer: the next generation. Cell.2011;144(5):646-674.

31. Baker DJ, Jin F, Jeganathan KB, van Deursen JM. Whole chromosome instability caused by Bub1 insufficiency drives tumorigenesis through tumor suppressor gene loss of heterozygosity. Cancer Cell. 2009;16(6):475-486.

32. Hagting A, Den Elzen N, Vodermaier HC, Waizenegger IC, Peters JM, Pines J. Human securin proteolysis is controlled by the spindle checkpoint and reveals when the APC/C switches from activation by Cdc20 to Cdh1. J Cell Biol. 2002;157(7):1125-1137.

33. Mardin BR, Agircan FG, Lange C, Schiebel E. Plk1 controls the Nek2A-PP1 $\gamma$ antagonism in centrosome disjunction. Curr Biol. 2011;21(13):1145-1151.

34. Zhang Y, et al. USP44 regulates centrosome positioning to prevent aneuploidy and suppress tumorigenesis. JClin Invest. 2012;122(12):4362-4374.

35. Garcia-Higuera I, et al. Genomic stability and tumour suppression by the APC/C cofactor Cdh1. Nat Cell Biol. 2008;10(7):802-811.

36. Lu LY, et al. Polo-like kinase 1 is essential for early embryonic development and tumor suppression. Mol Cell Biol. 2008;28(22):6870-6876.

37. Chesnokova V, Kovacs K, Castro AV, Zonis S, Melmed S. Pituitary hypoplasia in $\mathrm{Pttg}^{-/}$mice is protective for $\mathrm{Rb}^{+/}$pituitary tumorigenesis. Mol Endocrinol. 2005;19(9):2371-2379.

38. Gordon DJ, Resio B, Pellman D. Causes and consequences of aneuploidy in cancer. Nat Rev Genet. 2012;13(3):189-203.

39. Sanjana NE, Cong L, Zhou Y, Cunniff MM, Feng G, Zhang F. A transcription activator-like effector toolbox for genome engineering. Nat Protoc. 2012;7(1):171-192.

40. Li M, et al. The adaptor protein of the anaphase promoting complex Cdh1 is essential in maintaining replicative lifespan and in learning and memory. Nat Cell Biol. 2008;10(9):1083-1089.

41. Wu X, Kasper LH, Mantcheva RT, Mantchev
GT, Springett MJ, van Deursen JM. Disruption of the FG nucleoporin NUP98 causes selective changes in nuclear pore complex stoichiometry and function. Proc Natl Acad Sci U S A. 2001;98(6):3191-3196.

42. Babu JR, Jeganathan KB, Baker DJ, Wu X, KangDecker N, van Deursen JM. Rae1 is an essential mitotic checkpoint regulator that cooperates with Bub3 to prevent chromosome missegregation. JCell Biol. 2003;160(3):341-353.

43. Mei J, Huang X, Zhang P. Securin is not required for cellular viability, but is required for normal growth of mouse embryonic fibroblasts. Curr Biol. 2001;11(15):1197-1201.

44. Kasper LH, Brindle PK, Schnabel CA, Pritchard CE, Cleary ML, van Deursen JM. CREB binding protein interacts with nucleoporin-specific FG repeats that activate transcription and mediate NUP98-HOXA9 oncogenicity. Mol Cell Biol. 1999;19(1):764-776.

45. Pritchard CE, Fornerod M, Kasper LH, van Deursen JM. RAE1 is a shuttling mRNA export factor that binds to a GLEBS-like NUP98 motif at the nuclear pore complex through multiple domains. JCell Biol. 1999;145(2):237-254.

46. Ricke RM, Jeganathan KB, Malureanu L, Harrison AM, van Deursen JM. Bub1 kinase activity drives error correction and mitotic checkpoint control but not tumor suppression. J Cell Biol. 2012;199(6):931-949.

47. Dawlaty MM, et al. Resolution of sister centromeres requires RanBP2-mediated SUMOylation of topoisomerase II $\alpha$. Cell. 2008;133(1):103-115.

48. Groth P, et al. Methylated DNA causes a physical block to replication forks independently of damage signalling, $\mathrm{O}(6)$-methylguanine or DNA single-strand breaks and results in DNA damage. JMol Biol. 2010;402(1):70-82.

49. Matsumoto T, Wang PY, Ma W, Sung HJ, Matoba S, Hwang PM. Polo-like kinases mediate cell survival in mitochondrial dysfunction. Proc Natl Acad Sci U S A. 2009;106(34):14542-14546.

50. Hamada M, et al. Ran-dependent docking of importin- $\beta$ to RanBP2/Nup358 filaments is essential for protein import and cell viability. JCell Biol. 2011;194(4):597-612. 